\title{
Upgrade for Phase II of the GERDA experiment
}

\author{
GERDA Collaboration 1,a
}

M. Agostini ${ }^{15}$, A. M. Bakalyarov ${ }^{13}$, M. Balata ${ }^{1}$, I. Barabanov ${ }^{11}$, L. Baudis ${ }^{19}$, C. Bauer ${ }^{7}$, E. Bellotti ${ }^{8,9}$, S. Belogurov ${ }^{11,12, b}$, S. T. Belyaev ${ }^{13, c}$, G. Benato ${ }^{19}$, A. Bettini ${ }^{16,17}$, L. Bezrukov ${ }^{11}$, T. Bode ${ }^{15}$, D. Borowicz ${ }^{5, d}$, V. Brudanin ${ }^{5}$, R. Brugnera ${ }^{16,17}$, A. Caldwell ${ }^{14}$, C. Cattadori ${ }^{9}$, A. Chernogorov ${ }^{12}$, V. D'Andrea ${ }^{1}$, E. V. Demidova ${ }^{12}$, N. Di Marco' ${ }^{1}$ A. Domula ${ }^{4}$, E. Doroshkevich ${ }^{11}$, V. Egorov ${ }^{5}$, R. Falkenstein ${ }^{18}$, N. Frodyma ${ }^{3}$, A. Gangapshev ${ }^{7}, 11$, A. Garfagnini 16,17, P. Grabmayr ${ }^{18, a}$, V. Gurentsov ${ }^{11}$, K. Gusev ${ }^{5,13,15}$, J. Hakenmüller ${ }^{7}$, A. Hegai ${ }^{18}$, M. Heisel ${ }^{7}$, S. Hemmer ${ }^{16,17}$, R. Hiller ${ }^{19}$, W. Hofmann ${ }^{7}$, M. Hult ${ }^{6}$, L. V. Inzhechik ${ }^{11, d, e}$, L. Ioannucci ${ }^{1}$, J. Janicskó Csáthy ${ }^{15, f}$, J. Jochum ${ }^{18}$, M. Junker ${ }^{1}$, V. Kazalov11, Y. Kermaïdic ${ }^{7}$, T. Kihm ${ }^{7}$, I. V. Kirpichnikov ${ }^{12}$, A. Kirsch ${ }^{7}$, A. Kish ${ }^{19}$, A. Klimenko ${ }^{5,7}$, R. KneißI ${ }^{14}$, K. T. Knöpfle ${ }^{7}$, O. Kochetov ${ }^{5}$, V. N. Kornoukhov ${ }^{11,12}$, V. V. Kuzminov ${ }^{11}$, M. Laubenstein ${ }^{1}$, A. Lazzaro ${ }^{15}$, V. I. Lebedev ${ }^{13, b}$, B. Lehnert ${ }^{4, g}$, M. Lindner ${ }^{7}$, I. Lippi ${ }^{17}$, A. Lubashevskiy ${ }^{5}$, B. Lubsandorzhiev ${ }^{11}$, G. Lutter $^{6}$, C. Macolino ${ }^{1, h}$, B. Majorovits ${ }^{14}$, W. Maneschg ${ }^{7}$, E. Medinaceli ${ }^{16,17}$, M. Miloradovic ${ }^{19}$, R. Mingazheva ${ }^{19}$, M. Misiaszek ${ }^{3}$, P. Moseev ${ }^{11}$, I. Nemchenok ${ }^{5}$, S. Nisi ${ }^{1}$, K. Panas ${ }^{3}$, L. Pandola ${ }^{2}$, K. Pelczar ${ }^{1}$, A. Pullia ${ }^{10}$, C. Ransom ${ }^{19}$, S. Riboldi ${ }^{10}$, N. Rumyantseva ${ }^{5,13}$, C. Sada ${ }^{16,17}$, F. Salamida ${ }^{1, i}$, M. Salathe ${ }^{\text {, }}$, C. Schmitt ${ }^{18}$, B. Schneider ${ }^{4}$, S. Schönert ${ }^{15}$, J. Schreiner ${ }^{7}$, A.-K. Schütz ${ }^{18}$, O. Schulz ${ }^{14}$, B. Schwingenheuer ${ }^{7}$, O. Selivanenko ${ }^{11}$, E. Shevchik ${ }^{5}$, M. Shirchenko ${ }^{5}$, H. Simgen ${ }^{7}$, A. Smolnikov ${ }^{5,7}$, L. Stanco ${ }^{17}$, L. Vanhoefer ${ }^{14}$, A. A. Vasenko ${ }^{12}$, A. Veresnikova ${ }^{11}$, K. von Sturm ${ }^{16,17}$, V. Wagner $^{7}$, A. Wegmann ${ }^{7}$, T. Wester ${ }^{4}$, C. Wiesinger ${ }^{15}$, M. Wojcik ${ }^{3}$, E. Yanovich ${ }^{11}$, I. Zhitnikov ${ }^{5}$, S. V. Zhukov ${ }^{13}$, D. Zinatulina ${ }^{5}$, A. J. Zsigmond ${ }^{14}$, K. Zuber ${ }^{4}$ G. Zuzel $^{3}$

${ }^{1}$ INFN Laboratori Nazionali del Gran Sasso and Gran Sasso Science Institute, Assergi, Italy

2 INFN Laboratori Nazionali del Sud, Catania, Italy

${ }^{3}$ Institute of Physics, Jagiellonian University, Kraków, Poland

${ }^{4}$ Institut für Kern- und Teilchenphysik, Technische Universität Dresden, Dresden, Germany

5 Joint Institute for Nuclear Research, Dubna, Russia

${ }^{6}$ European Commission, JRC-Geel, Geel, Belgium

${ }^{7}$ Max-Planck-Institut für Kernphysik, Heidelberg, Germany

${ }^{8}$ Dipartimento di Fisica, Università Milano Bicocca, Milan, Italy

${ }^{9}$ INFN Milano Bicocca, Milan, Italy

${ }^{10}$ Dipartimento di Fisica, Università degli Studi di Milano e INFN Milano, Milan, Italy

${ }^{11}$ Institute for Nuclear Research of the Russian Academy of Sciences, Moscow, Russia

12 Institute for Theoretical and Experimental Physics, NRC "Kurchatov Institute", Moscow, Russia

13 National Research Centre "Kurchatov Institute", Moscow, Russia

${ }^{14}$ Max-Planck-Institut für Physik, Munich, Germany

${ }^{15}$ Physik Department and Excellence Cluster Universe, Technische Universität München, Munich, Germany

16 Dipartimento di Fisica e Astronomia dell'Università di Padova, Padua, Italy

17 INFN Padova, Padua, Italy

18 Physikalisches Institut, Eberhard Karls Universität Tübingen, Tübingen, Germany

${ }^{19}$ Physik Institut der Universität Zürich, Zurich, Switzerland

\begin{abstract}
The GERDA collaboration is performing a sensitive search for neutrinoless double beta decay of ${ }^{76} \mathrm{Ge}$ at the INFN Laboratori Nazionali del Gran Sasso, Italy. The upgrade of the GERDA experiment from Phase I to Phase II has been concluded in December 2015. The first Phase II data release shows that the goal to suppress the background
\end{abstract}

a e-mail: grabmayr@uni-tuebingen.de by one order of magnitude compared to Phase I has been achieved. GERDA is thus the first experiment that will remain "background-free" up to its design exposure (100 kg year). It will reach thereby a half-life sensitivity of more than $10^{26}$ year within 3 years of data collection. This paper describes in detail the modifications and improvements of the experimental setup for Phase II and discusses the performance of individual detector components. 


\section{Introduction}

Neutrinoless double beta $(0 \nu \beta \beta)$ decay is a hypothetical lepton number violating process, $(\mathrm{A}, \mathrm{Z}) \rightarrow(\mathrm{A}, \mathrm{Z}+2)+2 \mathrm{e}^{-}$, where inside a nucleus two neutrons convert into two protons and two electrons. Its observation would establish the neutrino to be its own anti-particle (Majorana particle), provide access to the absolute mass scale of neutrinos, and support extensions of the Standard Model of particle physics which try to explain the dominance of baryonic matter over antimatter in our universe [1]. Recent experiments have established the half-life of $0 \nu \beta \beta$ decay to be larger than $10^{25}$ year [2-4], and hence its detection requires the utmost suppression of any kind of background.

The GERmanium Detector Array (GERDA) collaboration searches at the INFN Laboratori Nazionali del Gran Sasso (LNGS) for $0 \nu \beta \beta$ decay of ${ }^{76} \mathrm{Ge},{ }^{76} \mathrm{Ge} \rightarrow{ }^{76} \mathrm{Se}+2 \mathrm{e}^{-}$. The GERDA experiment has been conceived in two phases. Here, a short overview of the experimental setup of Phase I which lasted from November 2011 until September 2013 is presented, while a detailed description has been given elsewhere [5].

The GERDA experiment is located underground below a rock overburden of about $3500 \mathrm{~m}$ water equivalent that eliminates the hadronic component of cosmic ray showers and reduces the muon flux to $\sim 1.25 /\left(\mathrm{m}^{2} \mathrm{~h}\right)$. GERDA uses high purity germanium (HPGe) detectors enriched in ${ }^{76} \mathrm{Ge}$ which are arranged in strings within a cryostat filled with $64 \mathrm{~m}^{3}$ of liquid argon (quality 5.0), see Fig. 1. The liquid argon (LAr) acts both as cooling and shielding medium. The cryostat itself is enclosed by a large tank containing $590 \mathrm{~m}^{3}$ of ultra-pure water produced by the BOREXINO water plant [6]; the $2-3 \mathrm{~m}$ thick water layer serves both as additional passive shield as well as the medium for a Cherenkov veto system with 66 photomultiplier tubes (PMTs) against muons. A clean room on top of the cryostat and water tank houses a glove box and the lock for assembly and deployment of the Ge detectors.

The Phase 1 detector array, 4 strings in total, consisted of 8 enriched semi-coaxial Ge detectors with a total mass of $15.6 \mathrm{~kg}$ and 3 semi-coaxial Ge detectors from lowbackground natural material. The one string of natural $\mathrm{Ge}$

a e-mail: gerda-eb@mpi-hd.mpg.de

b INFN Laboratori Nazionali del Gran Sasso, Italy

${ }^{c}$ Also with NRNU MEPhI, Moscow, Russia

$\mathrm{d}$ deceased

e Also with The Henryk Niewodniczanski Institute of Nuclear Physics PAS, Krakow, Poland

${ }^{f}$ Also with Moscow Institute for Physics and Technology, Moscow, Russia

g Now at IKZ, Dresden, Germany

h Now at Carleton University, Ottawa, Canada

${ }^{i}$ Now at LAL, CNRS/IN2P3, Université Paris-Saclay, Orsay, France

j Also with Dipartimento di Scienze Fisiche e Chimiche, Università degli Studi dell'Aquila, L'Aquila, Italy

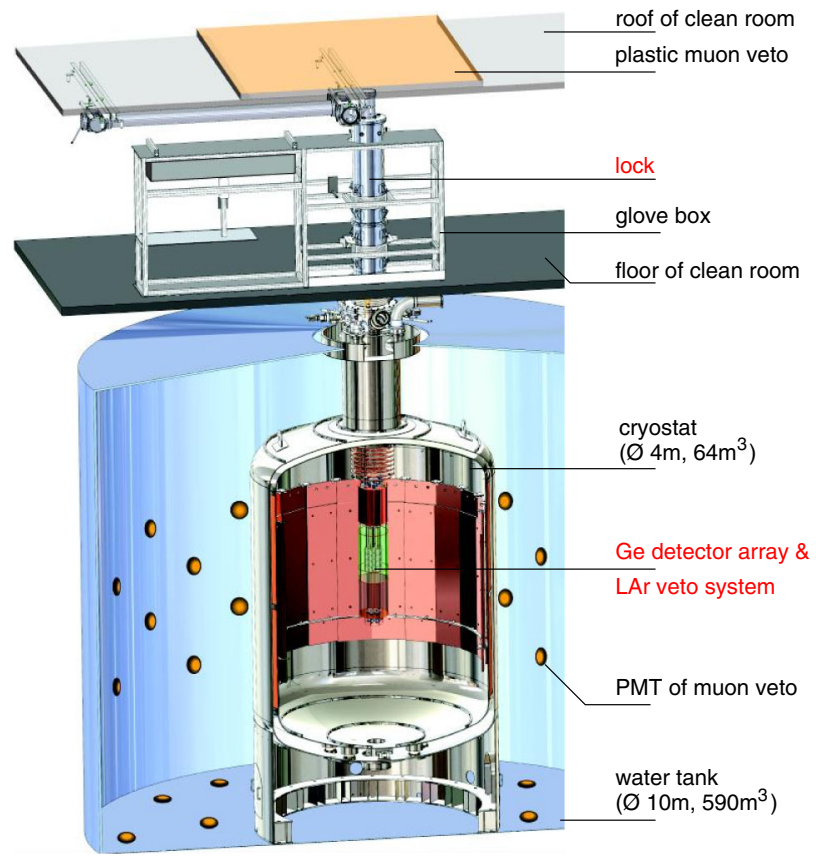

Fig. 1 GERDA setup. The new Phase II components are labeled in red

detectors was replaced in July 2012 by 5 Broad Energy Germanium (BEGe) detectors with a total mass of $3.6 \mathrm{~kg}$; these diodes served as prototypes for Phase II.

The physics results of Phase I [7] were based on an exposure of $21.6 \mathrm{~kg}$ year. The energy scale was determined by (bi)weekly calibrations with ${ }^{228} \mathrm{Th}$ sources. In the region of interest (ROI) around $\mathrm{Q}_{\beta \beta}=2039 \mathrm{keV}$, the interpolated exposure-averaged energy resolution of the enriched semicoaxial and BEGe detectors was determined to be $4.8(2) \mathrm{keV}$ and $3.2(2) \mathrm{keV}$ in terms of full-width-at-half-maximum (FWHM), respectively. A background index (BI) of about $10^{-2} \mathrm{cts} /(\mathrm{keV} \mathrm{kg}$ year) was achieved, one order of magnitude lower than in the best previous $0 \nu \beta \beta$ decay searches with ${ }^{76} \mathrm{Ge}$. No signal was found for $0 \nu \beta \beta$ decay, and a new $90 \%$ confidence level (CL) limit of $T_{1 / 2}^{0 \nu}>2.1 \times 10^{25}$ year was derived (median sensitivity $2.4 \times 10^{25}$ year) that strongly disfavored a previous claim of observation $[8,9]$. Further Phase I results include a much improved half-life for $2 \nu \beta \beta$ decay of ${ }^{76} \mathrm{Ge}$ and improved limits for Majoron $\beta \beta$ decay modes [10], as well as $2 \nu \beta \beta$ decays of ${ }^{76} \mathrm{Ge}$ into excited states of ${ }^{76} \mathrm{Se}[11]$.

Phase II of GERDA was designed to improve the sensitivity on the half-life of $0 v \beta \beta$ decay by about one order of magnitude. At the end of Phase I GERDA had left the "backgroundfree" regime ${ }^{1}$ where sensitivity scales linearly with exposure $\mathcal{E}=\mathrm{M} \mathrm{t}$, the product of detector mass $\mathrm{M}$ and measurement period $\mathrm{t}$, and entered the background i.e. statistical fluctua-

\footnotetext{
1 The "background-free" regime is effective if the background contribution is less than 1 in the energy region of interest $\left(Q_{\beta \beta} \pm 0.5 \mathrm{FWHM}\right)$.
} 


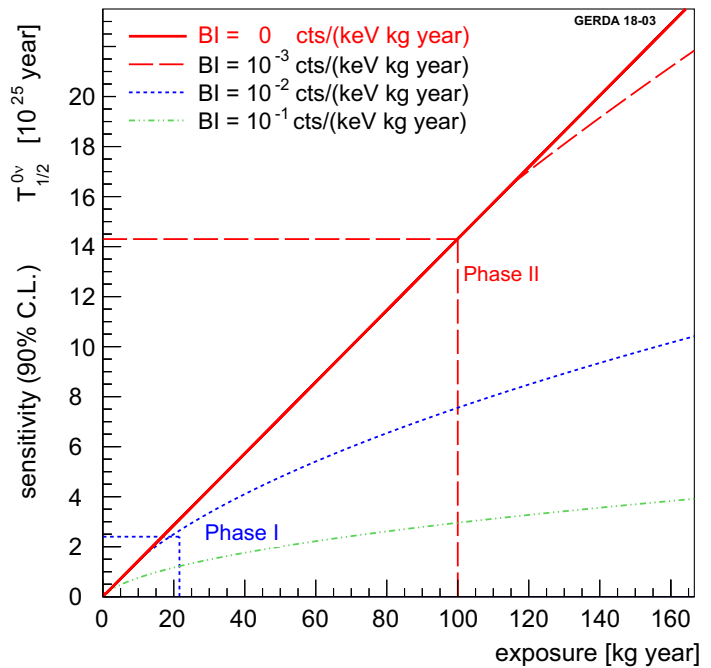

Fig. 2 Frequentist's estimate of the sensitivity of the GERDA experiment as a function of exposure for various background indices. An overall detection efficiency of $60 \%$ is assumed. The scenarios for GERDA Phase I and II are indicated

tion limited scenario where it scales approximately with the square root of exposure divided by the background index $\mathrm{BI}$ and the energy resolution $\Delta \mathrm{E}, \sqrt{(\mathcal{E} /(B I \cdot \Delta E)}$. An efficient upgrade requires thus to re-enter the "background-free" regime, that means to not only increase exposure (detector mass) but also to reduce correspondingly the background (see Fig. 2). Improvements of the already excellent resolution are possible but limited to a factor of about 2 for the given technology. GERDA thus needs to achieve a BI of $10^{-3} \mathrm{cts} /(\mathrm{keV} \mathrm{kg}$ year) in Phase II in order to reach the desired sensitivity beyond $10^{26}$ year at an exposure of about $100 \mathrm{~kg}$ year (see Fig. 2).

The analysis of the Phase I data showed that most background events were due to radioactive isotopes in materials close to the detectors [12]. The straightforward consequence was to further reduce material close to the detectors and/or to replace it by material of higher radiopurity. The major BI reduction had to come, however, from a largely improved discrimination of background events taking full advantage of their different event topology. While $0 \nu \beta \beta$ events normally deposit energy in a confined volume (a few $\mathrm{mm}^{3}$ ) of the detector, the background events can also deposit energy in the LAr around the detector, at the detector surface, or scatter at several locations in the detector. Events can thus be identified as background by coincident scintillation light in the LAr, by coincidences within the detector array and/or by the analysis of the signal pulse shape. GERDA has taken full advantage of all these options in Phase II: the additional batch of $20 \mathrm{~kg}$ of enriched Ge detectors consists of diodes of the novel BEGe type exhibiting superior pulse shape discrimination (PSD) [13] and energy resolution; a larger and more densely packed detector array exhibits enhanced efficiency for detector-detector (anti-)coincidences, and importantly, the LAr around the detector array has been instrumented for the readout of scintillation light creating thus an effective active LAr veto system. The efficacy of this approach has indeed been proven by the first results obtained with the upgraded GERDA experiment. Started in December 2015, the Phase II physics run reached in June 2016 the exposure of $10.8 \mathrm{~kg}$ year. These accumulated data have been already sufficient to demonstrate that the projected background level of $10^{-3} \mathrm{cts} /(\mathrm{keV} \mathrm{kg}$ year) has been achieved and, to extract in combination with the Phase I data set a new lower limit for the $0 v \beta \beta$ decay half-life of ${ }^{76} \mathrm{Ge}$ of $>5.3 \times 10^{25}$ year at $90 \%$ CL [3].

The following sections describe the modifications of the GERDA experimental setup for Phase II including the new detector components and their performance. Section 2 presents an overview of the properties of the coaxial and BEGe detectors making up the Phase II detector array; it provides also details of the new mechanical mounts, cabling, electrical contacts and the cold electronic front end. A major part of this paper, Sect. 3, is devoted to the LAr veto system. Section 4 discusses the modifications of the infrastructure, in particular the new lock needed for the largely increased detector array and the LAr veto system. Section 5 summarizes the screening results for the newly introduced components. The performance of the individual subsystems and the background level achieved in Phase II until April 2017 are presented in Sect. 6. Conclusions are given in Sect. 7.

\section{Germanium detectors}

\subsection{Characteristics of Phase II detectors}

The GERDA Phase II detector array includes 7 strings, which carry 40 detectors in total. The detectors can be divided into three groups: the newly produced BEGe detectors, the semicoaxial ANG and RG detectors, and the semi-coaxial GTF detectors [5]. The detectors of the first two groups are made of germanium enriched in ${ }^{76} \mathrm{Ge}$ ( ${ }^{\text {enr }} \mathrm{BEGe}$, ${ }^{\text {enr }} \mathrm{Coax}$ ), while those of the third group are made of germanium with natural isotopic abundance ( $\left.{ }^{\text {nat }} C o a x\right)$. The main properties of the individual detectors groups are discussed below, the properties of the individual detectors are listed in the Appendix (see Table 14).

\subsubsection{The semi-coaxial detectors}

The 7 semi-coaxial ${ }^{76} \mathrm{Ge}$ enriched detectors (ANG, RG), which originated from the former Heidelberg-Moscow and IGEX experiments, represented the core of GERDA Phase I [5]. In GERDA Phase II, they have again been included. 
The ${ }^{76}$ Ge enrichment fractions of the ANG and RG detectors are in the range of $85.5-88.3 \%$. All ${ }^{\text {enr Coax detectors }}$ have masses greater than $2 \mathrm{~kg}$, except ANG1. The total mass is $15.578(7) \mathrm{kg}$. The applied bias voltages coincide nearly with those $V_{\text {rec }}$ recommended by the manufacturer [14]. The ${ }^{76} \mathrm{Ge}$ content of the 3 natural GTF detectors corresponds to the natural abundance of $7.8 \%$.

\subsubsection{The BEGe detectors}

In order to increase the exposure (via increase of total detector mass) and to improve the background index (via an enhanced pulse shape performance), the GERDA collaboration opted for the production of 30 new detectors following the BEGe design of the company Canberra [15]. After extensive preparation, 30 detectors were delivered. Only one detector, GD02D, turned out to be rather a p-n junction than of p-type material, and thus suitable only for anti-coincidence studies in GERDA.

The 30 enr BEGe detectors have an enrichment fraction of $87.8 \%$. The diameters of the detectors range from $58.3(1) \mathrm{mm}$ to 79.0(1) $\mathrm{mm}$, and their heights from 22.9(3) to $35.3(1) \mathrm{mm}$. In 21 cases the detectors are cylindric, in 9 cases they have a conical shape. The latter shape was tolerated in order to maximize the number of crystal slices that can be obtained from one single crystal ingot. The total mass is $20.024(30) \mathrm{kg}$. Herein, the $\pm 1 \mathrm{~g}$ error from weighing was assumed to be correlated for all detectors. Neglecting the detector GD02D, the total detector mass is reduced to $19.362(29) \mathrm{kg}$.

The average active volume fraction $f_{a v}$ and the total active mass $M_{a v}$ of all 29 fully operational GERDA Phase II BEGe detectors have been determined combining the full charge collection depth (FCCD) results from ${ }^{241} \mathrm{Am}$ and ${ }^{133} \mathrm{Ba}$ source measurements conducted in the HADES underground laboratory [16]. Moreover, an increase of the FCCD of 0.2 to $0.3 \mathrm{~mm}$ due to storage at room temperature over a period of nearly 3 years has been considered. All in all this led to:

$f_{a v}=0.885_{-0.015}^{+0.016}$ (uncorr) ${ }_{-0.003}^{+0.006}$ (corr)

$M_{a v}=17.132_{-0.294}^{+0.315}$ (uncorr) ${ }_{-0.063}^{+0.123}$ (corr) $\mathrm{kg}$.

Compared to the initial purified Ge powder used for crystal pulling, a crystal mass yield of $50.1 \%$ and an active mass yield of $48.2 \%$ were achieved (see Table 1). Considering that approximately $25 \%$ of the kerf material could be recovered, the achieved total mass yield is high.

The bias voltages applied on the detectors in GERDA coincide typically with the values $V_{\text {rec }}$ recommended by the manufacturer (see "Appendix" Table 14). The collaboration, however, performed detailed voltage scans, in which depletion voltages and new operational voltages were determined which still guarantee uncompromised detector response. The new values are $600 \mathrm{~V}$ lower, on average. Indeed, GERDA oper-
Table 1 Crystal and active mass yield in \% from enriched germanium to the final 30 BEGe detectors for GERDA Phase II. The mass transfer fractions are given relative to the original enriched $\mathrm{GeO}_{2}$ material ( $3 \mathrm{rd}$ column) and to the purified metallic Ge used for crystal growth (4th column)

\begin{tabular}{llcl}
\hline Germanium operation & Mass $(\mathrm{kg})$ & \multicolumn{2}{c}{ Relative fraction } \\
\cline { 3 - 4 } & & $(\%)$ & $(\%)$ \\
\hline Ge in $\mathrm{GeO}_{2}$ after enrichment & 37.5 & 100.0 & - \\
Purified Ge for crystal growth & 35.5 & 94.1 & 100.0 \\
30 produced diodes & 20.0 & 53.3 & 56.3 \\
29 operational diodes & 19.4 & 51.7 & 54.6 \\
Active mass of 29 diodes & 17.1 & 45.6 & 48.2 \\
\hline
\end{tabular}

ates in a few cases the detectors at these lower values, preventing thus unwanted high leakage currents or other instabilities.

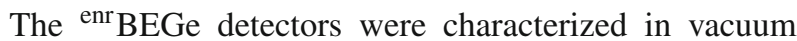
cryostats within the HADES underground laboratory [16]. Note that these measurements were performed with 'passivated' detectors where the groove between the $\mathrm{p}^{+}$and $\mathrm{n}^{+}$electrode is covered by an insulating silicon monoxide ('passivation') layer [15]. The energy resolution of all detectors turned out to be excellent. At the $1333 \mathrm{keV}{ }^{60} \mathrm{Co} \gamma$-line the FWHM energy resolution is $1.72(7) \mathrm{keV}$, with best and worst values of $1.59 \mathrm{keV}$ (GD89A) and $1.87 \mathrm{keV}$ (GD79C). While a small dependence on the detector mass became visible, no dependence on the detector shape could be observed, i.e. cylindric and conical ones have the same performance.

The pulse shape discrimination power was deduced from ${ }^{228} \mathrm{Th}$ source measurements. For an event selection criterion keeping $90 \%$ of signal-like events (double-escape peak events from the ${ }^{208} \mathrm{Tl}$ line), the following survival probabilities for background-like event populations were found: the single-escape peak is reduced to (5-12)\%, the full-energy peaks at 2615 and $1620 \mathrm{keV}$ survive at (6-19)\% and (9-19)\%, respectively, and the Compton-events in the ROI are in the range of $(32-48) \%$.

\subsubsection{Electrical contacts}

Aluminum bonding pads of $600 \mathrm{~nm}$ thickness have been evaporated on the $\mathrm{p}^{+}$signal and $\mathrm{n}^{+}$high-voltage electrodes of all Phase II detectors. The evaporation process had been developed in collaboration with the company Canberra and subsequently integrated together with the dedicated evaporation system into the manufacturer's production chain [17]. 


\subsubsection{Activation by cosmic rays}

Great attention has been paid to minimize the activation of the newly produced BEGe detectors by cosmic rays [15]. As of January 1 2017, we expect from cosmic activation in the 30 BEGes detectors $(20.02 \mathrm{~kg})$ about $24{ }^{68} \mathrm{Ge}$ nuclei and $300{ }^{60} \mathrm{Co}$ nuclei; ${ }^{2}$ for comparison, the saturation activity is $\sim 2300{ }^{68} \mathrm{Ge}$ nuclei and $\sim 9200{ }^{60} \mathrm{Co}$ nuclei per kg germanium, respectively. Simulations show that during the year 2017 the decay of these nuclei will increase the background index before LAr veto and PSD by $2.5 \times$ $10^{-4} \mathrm{cts} /\left(\mathrm{keV} \mathrm{kg}\right.$ year) and $0.34 \times 10^{-4} \mathrm{cts} /(\mathrm{keV} \mathrm{kg}$ year $)$, respectively. For the semi-coaxial enriched detectors, the ${ }^{68} \mathrm{Ge}$ activation has vanished due to their long underground storage; the ${ }^{60} \mathrm{Co}$ contribution is about $1 / 3$ of the expectation for the BEGe detectors.

\subsection{Detector mount and cabling}

The GERDA Phase I background has been shown to originate predominantly from sources close to the Ge detectors. In addition, since the average mass ratio of BEGe to semicoaxial detectors is about 1:3, further optimization of the detector mount for Phase II required to reduce the amount of construction materials and/or to improve their radiopurity. Figure 3 shows the original Phase II BEGe detector module which consists of 2 BEGe Ge diodes that are mounted back-to-back. The new design replaces the Phase I spring loaded contacts to the detector electrodes by $25 \mu \mathrm{m}$ diameter $\mathrm{Al}$ wire bonds. This allowed the substitution of a large part of the Phase I copper material (and PTFE) by mono-crystalline silicon which is less strong but intrinsically extremely radiopure (see Tables 2 and 8). The silicon plate serves both to define the position of the vertical copper bars which take the weight of the Ge detectors and to provide the substrate onto which signal and high voltage cables are attached with bronze clamps. The top and bottom of the copper bars carry bolts and nuts for the connection to another detector module.

Another advantage of the new holder is that the detector mounting procedure becomes easier and safer than in Phase I, since all mounting steps except bonding are being done without touching the diode. Thus the possibility to scratch the very sensitive $\mathrm{p}^{+}$contact is minimized. Some detectors have been mounted and dismounted in their Phase II holders several times without any deterioration of their performance. Bonding also solved the previous problems of irreproducible $\mathrm{HV}$ contact quality.

During commissioning for Phase II, so far unknown problems with detector biasing and leakage currents showed

\footnotetext{
${ }^{2}$ Activation rates at sea level vary from 1 to 13 and 1.6 to 6.7 nuclei/(kg day) for ${ }^{68} \mathrm{Ge}$ and ${ }^{60} \mathrm{Co}$ nuclei, respectively. Here, we assume 5.8 and 3.3 nuclei/(kg day) [15].
}
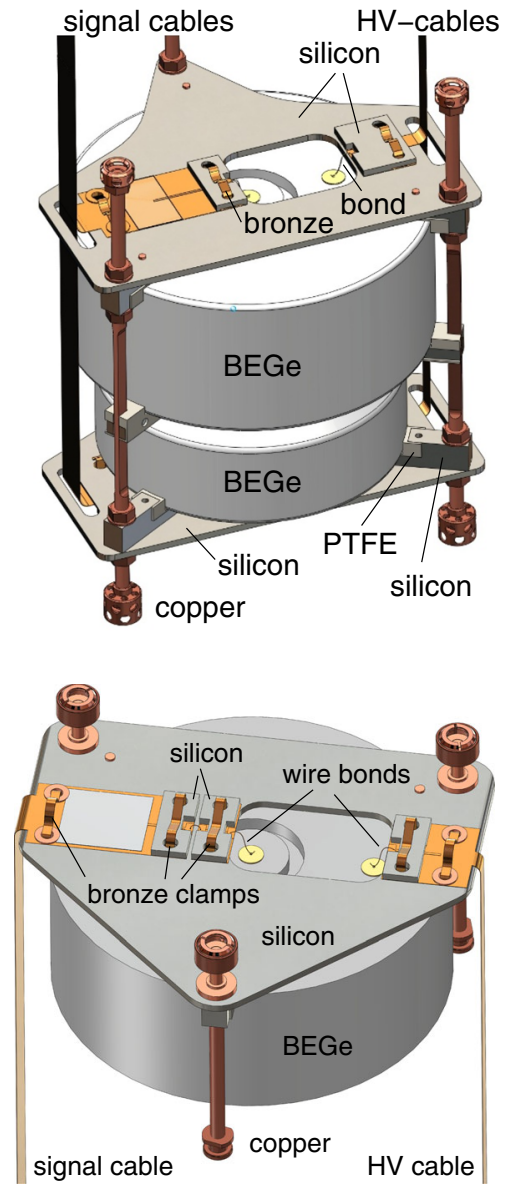

Fig. 3 Alternative mounts for pairs of and single BEGe detectors, and their connection to the signal and high voltage (HV) flexible cables. The single detector module is viewed from bottom

Table 2 Comparison of the masses $m$ of construction materials for the Phase I and II detector holders, and of the masses $m^{\prime}$ normalized to $1 \mathrm{~kg}$ of detector mass assuming an average mass of $0.67 \mathrm{~kg}$ for single BEGe and $2.4 \mathrm{~kg}$ for semi-coaxial detectors

\begin{tabular}{llllll}
\hline Material & \multicolumn{2}{l}{ Phase I (coaxial) } & & \multicolumn{2}{l}{ Phase II (BEGe) } \\
\cline { 2 - 3 } \cline { 6 - 6 } & $m(\mathrm{~g})$ & $m^{\prime}(\mathrm{g} / \mathrm{kg})$ & & $m(\mathrm{~g})$ & $m^{\prime}(\mathrm{g} / \mathrm{kg})$ \\
\hline $\mathrm{Cu}$ & 84 & 35.0 & & 13 & 19.4 \\
$\mathrm{Si}$ & 1 & 0.4 & & 20 & 29.9 \\
PTFE & 7 & 2.9 & & 1 & 1.5 \\
CuSn6 & - & - & & 0.7 & 0.97 \\
Total & 92 & 38.3 & & 34.7 & 51.8 \\
\hline
\end{tabular}

up that were highly correlated with the orientation of BEGe detectors such that detectors with the groove pointing upwards, the 'top' detectors in the mount, were much more affected than the 'bottom' detectors with the groove pointing downwards. In Phase I all detectors had been indeed mounted with the groove pointing downwards. The problem could be attributed to microscopic particulates which had fallen into 


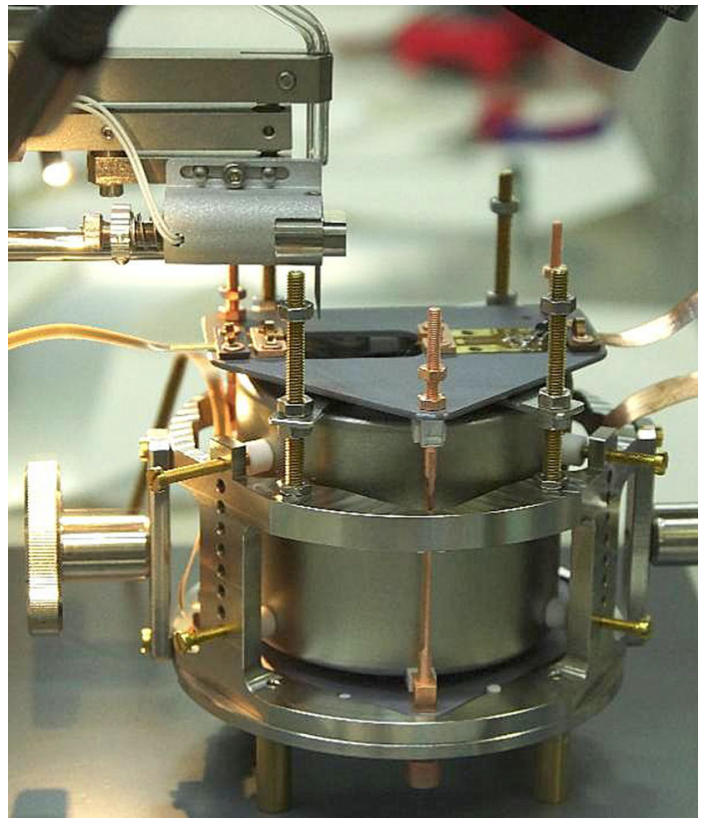

Fig. 4 A detector module with two BEGe detectors within its jig. The top plate of the jig has been removed to provide full access for the deep-access bonder

the groove during the mounting procedure or during operation in the LAr. Hence, the concept of mounting pairs of BEGe detectors was given up, and single BEGe detectors were mounted individually like the semi-coaxial detectors avoiding grooves pointing upwards (see Fig. 3, bottom). By the start of the Phase II physics run, all problematic BEGe pair assemblies and also the newly deployed detectors received the new holders which virtually eliminated the previous problems. It is planned to replace the mount of the 6 remaining BEGe pair assemblies in a forthcoming maintenance break.

The low-mass Phase II detector mount with its silicon plate has no tolerance against crack-producing stress, thus requires the detector assembly to be kept in a jig until finally deployed in the detector string. The rather compact jig is made from stainless steel and thoroughly cleaned in order to avoid contamination of the detector module. Figure 4 shows a jig and detector module within a glove box, flushed with nitrogen, in front of a deep-access bonder ready for connecting the flexible cables with the signal and HV contact of the top BEGe detector. After turning the jig for bonding the second detector, jig and module are stored in their dedicated vacuum container until deployment.

After mounting and bonding, each detector was tested for leakage current in the GERDA Germanium Detector Laboratory (GDL) which is also located underground at LNGS. All detectors that passed this test were integrated in the GERDA setup. However, about half of the diodes showed again high leakage currents already in the GDL test bench. They were dismounted from their holders and sent to Canberra for repro-

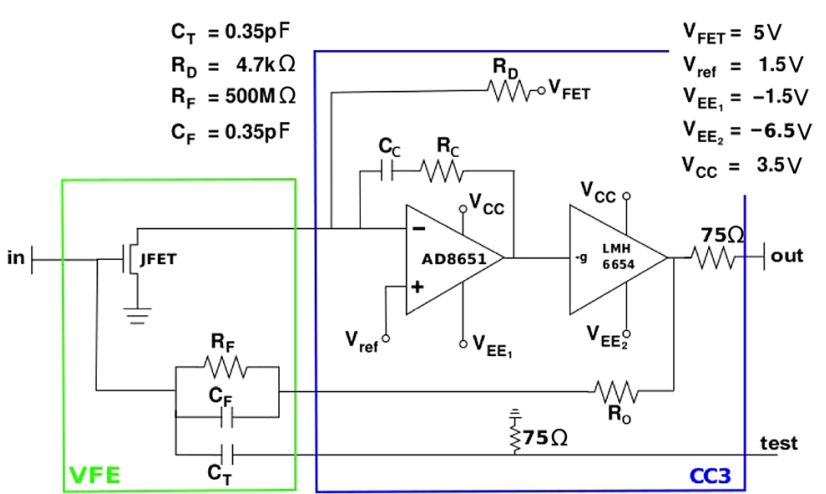

Fig. 5 Schematic of one channel of the $\mathrm{CC} 3 \mathrm{Ge}$ readout circuit. The green frame shows the very front end (JFET, feedback resistor and capacitor), the blue frame the following stages

cessing and, in some cases, passivation. On return they were mounted again in the holders, tested in GDL and added to the GERDA setup at the final stage of integration.

\subsection{Ge detector readout}

The Ge detectors are read out with custom-produced preamplifiers called ' $\mathrm{CC} 3$ '. Figure 5 shows the schematic of this cryogenic, low radioactivity, 4-channel charge sensitive amplifier $[18,19]$. It is a continuous resistor reset amplifier, made of two amplifying stages based on commercial CMOS operational amplifiers. Miniaturized SMD components and Ta capacitors, mostly in 0402 size, are chosen to minimize the radioactivity of the circuit (Fig. 6). The input stage is the BF862 JFET from NPX. The CC 3 circuit has a sensitivity of $\sim 150 \mathrm{mV} / \mathrm{MeV}$, a dynamic range of $\sim 15 \mathrm{MeV}$, a rise-time $(10-90 \%)$ of $<100 \mathrm{~ns},<70 \mathrm{~mW} / \mathrm{ch}$ power consumption, and an intrinsic noise of $\sim 0.8 \mathrm{keV}$ Ge-equivalent.

The Ge readout electrode is connected to the JFET-PCB by a flexible flat cable (FFC), made from Pyralux ${ }^{\circledR}$ or Cuflon ${ }^{\circledR}$. This allows for a detachable contact between the detector and the main front end board, without any glueing or soldering at the detector level, hence minimizing the activity close to the detectors while maximizing the contact reliability. Two different FFCs are adopted for the signal and HV contact: the HV FFCs are made from 10 mils Cuflon ${ }^{\circledR}$, or 3 mils Pyralux ${ }^{\circledR}$, the signal FFCs from 3 mils Cuflon ${ }^{\circledR}$ or Pyralux ${ }^{\circledR}$. For all FFCs the $\mathrm{Cu}$ trace is $2 \mathrm{~mm}$ wide and $18 \mu \mathrm{m}$ thick.

\subsection{The seven detector strings}

Figure 7 shows the Ge detector array together with the electronic front end boards on top in about $30 \mathrm{~cm}$ distance. The height of the array is $40 \mathrm{~cm}$, its diameter is about $30 \mathrm{~cm}$. There are 7 strings with 40 detectors in total. Six strings consist either of $8 \mathrm{BEGe}$ or 3 semi-coaxial (enriched or natural) detectors. One string is a mixture of semi-coaxial and BEGe 


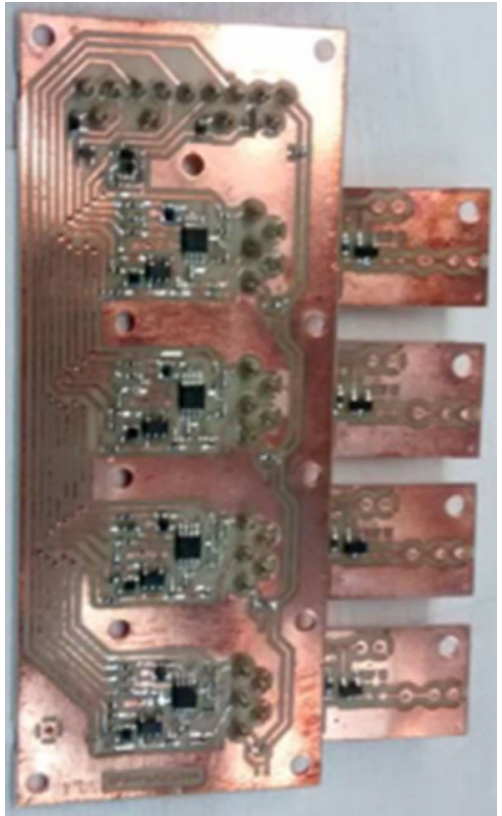

Fig. 6 Photo of the $\mathrm{CC} 3 \mathrm{Ge} 4$-channel readout circuit. Each circuit serves 4 channels. The very front end components (see Fig. 5) are located on the 4 small PCBs which are connected with low activity pin-contacts to the main board; this allows us to replace individually for each channel the components of the very front end, and in particular the rather sensitive JFET

detectors. A photo of the detector array is shown in Fig. 31 (see Appendix).

Further figures in the Appendix show the array from the top including the location of the calibration sources as well as the inner boundary of the LAr veto system (Fig. 32) and the detailed arrangement of all detectors (Fig. 33). Detectors marked with blue are passivated. It should be noted that, contrary to Phase I experience, no leakage current increase has been found for neither passivated nor non-passivated diodes after one year of operation (see Sect. 6 and Fig. 20).

\subsection{The mini-shrouds for mitigating ${ }^{42} \mathrm{~K}$ background}

The background due to ${ }^{42} \mathrm{Ar}$ is prominent in GERDA. ${ }^{42} \mathrm{Ar}$ decays into ${ }^{42} \mathrm{~K}$, which is a $\beta$ emitter with an endpoint energy of $3.5 \mathrm{MeV}$. A copper cylinder, called 'mini-shroud' (MS), placed around the detectors was used for mitigation of ${ }^{42} \mathrm{~K}$ background in Phase I [5]. The MS screens the electric field of the detector and creates a mechanical barrier which prevents the collection of ${ }^{42} \mathrm{~K}$ ions on the detector surface. The volume from which ${ }^{42} \mathrm{~K}$ collection takes place becomes much smaller and thus the level of ${ }^{42} \mathrm{~K}$ background decreases. However, the copper MS cannot be used in GERDA Phase II since the LAr scintillation veto is implemented for the suppression of various backgrounds. Scintillation light generated inside the copper MS would not be visible by the LAr instrumentation and the efficiency of the LAr veto system would be

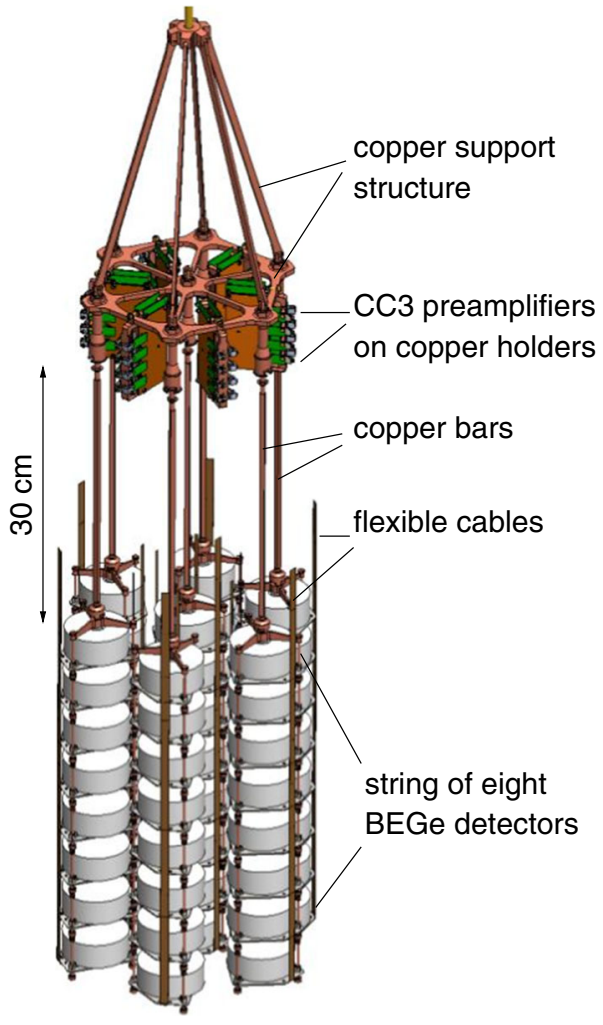

Fig. 7 Arrangement of the seven detector strings with preamplifiers

considerably reduced. Another reason for the development of a new MS is the higher demands on radiopurity which would not have been met by the copper MS of Phase I.

That is why for Phase II a new MS made from ultra-pure nylon was developed [20]. A photo of the detector array with each string enclosed by its individual transparent MS is shown in Fig. 31 of the "Appendix". Such a nylon MS does not screen the electric field of the detector like a copper one, but serves just as a barrier that stops the drift of ${ }^{42} \mathrm{~K}$ ions towards the detectors. The nylon films were provided by Princeton University. They were fabricated for the BOREXINO internal balloon [21]. The thickness of the films is $125 \mu \mathrm{m}$. Similar to other plastics materials, nylon is almost opaque for the deep ultraviolet radiation generated in LAr. Hence it is covered on both sides with a wavelength shifter (WLS) based on tetra-phenyl-butadiene (TPB), that shifts the $128 \mathrm{~nm}$ scintillation light to wavelengths of about $450 \mathrm{~nm}$, suitable for transport through the nylon and detection by the LAr veto system.

The investigation and development of the background suppression methods were done at the low-background test facility LARGE [22] in GDL. It was demonstrated that the nylon MS is robust enough to be deployed into the cryogenic liquid and that it does not deteriorate the LAr scintillation veto performance. By reducing the collection of ${ }^{42} \mathrm{~K}$ atoms at the surface of the Ge detector, and by combining the 


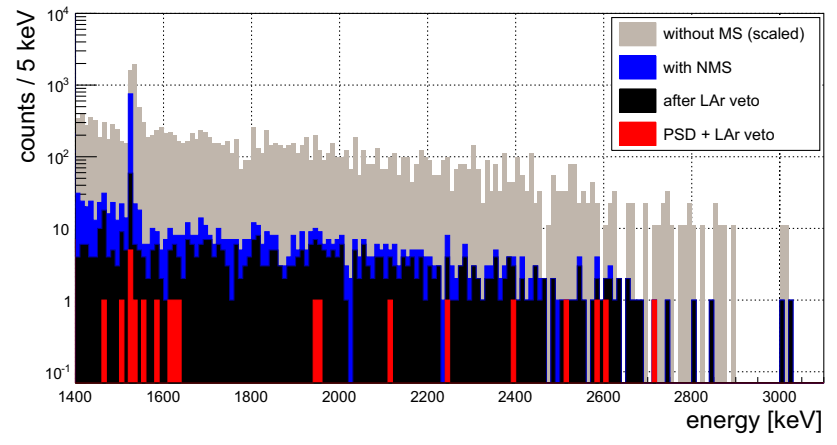

Fig. 8 Suppression of the events from ${ }^{42} \mathrm{Ar}$ decays by the nylon minishroud (NMS), LAr veto and PSD: the grey area shows the scaled spectrum from a measurement with a bare BEGe detector without NMS, 'blue' the measurement with NMS, 'black' the events surviving the LAr veto cut, and 'red' the remaining events after both the LAr veto and PSD cuts [20]

PSD technique with the scintillation veto, it was possible to decrease the ${ }^{42} \mathrm{~K}$ background by more than a factor of $10^{3}$ (see Fig. 8).

\section{LAr veto system}

The liquid argon veto system (LAr veto) of GERDA is a detector system devised to detect argon scintillation light in the vicinity of the Ge detector array. It evolved from studies of scintillation light detection in LAr with 8" PMTs in the low-background facility LARGE [22] and silicon photomultipliers (SiPMs) coupled to wave length shifting fibers for increasing light detection efficiency [23]. The goal is to reject those types of background events in the Ge detectors that simultaneously deposit energy in the surrounding LAr, and hence generate scintillation. These background types mainly include $\gamma$-ray background from $\mathrm{Ra}$ and Th decays in solid materials inside and around the detectors. But also other types of background can successfully be rejected, such as muons or decays from ${ }^{42} \mathrm{Ar} /{ }^{42} \mathrm{~K}$. The concept of a LAr anticoincidence veto has been proven in LARGE where suppression factors of up to a few times $10^{3}$ were achieved depending on the background type and source distance towards the $\mathrm{Ge}$ detectors [22].

The LAr light instrumentation in GERDA is conceived as a hybrid system comprising PMTs and WLS fibers with SiPM readout. It is designed to be a retractable unit that can be deployed together with the Ge detector array into the cryostat through the lock system. The elaborated mounting scheme of the veto system is described in the next section. The lock system constrains the veto's geometry to a lengthy cylindrical shape with a diameter of $\sim 0.50 \mathrm{~m}$ and a total height of $\sim 2.6 \mathrm{~m}$. A (CAD) technical drawing depicting the complete LAr veto system is shown in Fig. 9.

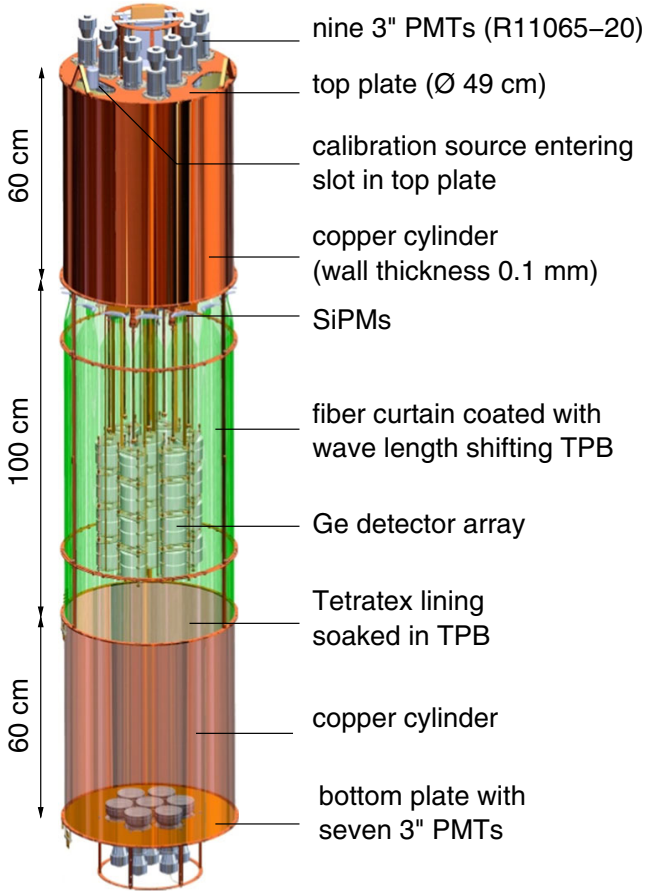

Fig. 9 The Ge detector array enclosed by the LAr veto system

\subsection{PMT system}

The PMT light readout system measures the scintillation light around the detector array with nine PMTs from the top and seven PMTs from the bottom. The 3" PMTs are installed on copper plates at the two ends of the cylindrical LAr volume facing inwards (see Figs. 10,11). These end plates are separated from the central fiber section by copper shrouds of $60 \mathrm{~cm}$ height. The copper shrouds consist of $100 \mu \mathrm{m}$ thick copper foils which carry laser-welded flanges at both ends for the connection to the PMT plates and the fiber section. They are lined with Tetratex ${ }^{\circledR}$ PTFE foil of $254 \mu \mathrm{m}$ thickness from the inside. The Tetratex ${ }^{\circledR}$ foil is impregnated with TPB, and thereby serves as a WLS of the scintillation and diffuse reflector of the shifted light. The foil has been thoroughly tested for mechanical and optical stability of the WLS [24].

The cabling of the bottom PMTs runs along the outside of the LAr veto system. The bottom shroud can be easily detached from the central fiber unit within the glove box. For this purpose custom made low-radioactivity cable plugs for the bottom PMTs were placed on the connecting flange (see Fig. 12).

The PMTs are of type Hamamatsu R11065-20 Mod. They have a bialkali photocathode that reaches a quantum efficiency of about $40 \%$ at the wavelength of $420 \mathrm{~nm}$. Typically a peak-to-valley ratio of about four is achieved for single photoelectrons. To enable direct detection of scintillation the photocathodes are coated with a 1-4 $\mu \mathrm{m}$ thick layer of WLS, TPB (10\% by mass) embedded in polystyrene (90\%). In vari- 


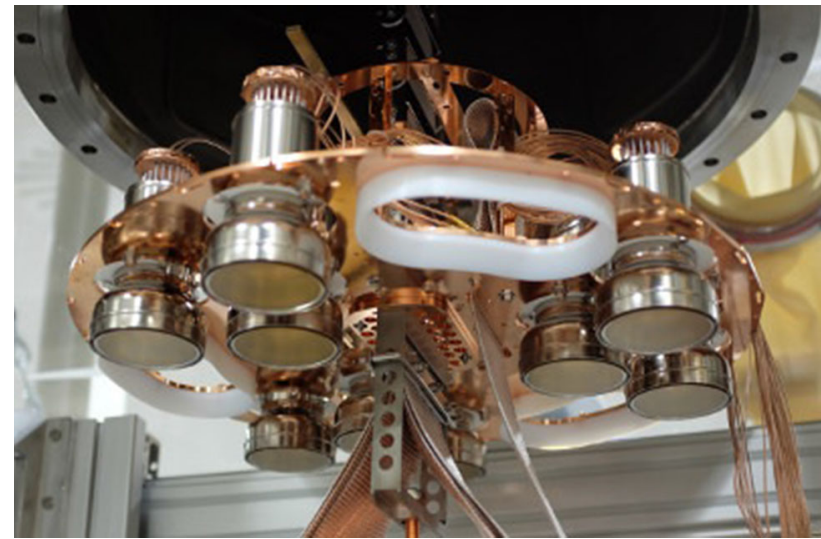

Fig. 10 Top PMT plate with 9 PMTs and three longitudinal slots with PTFE guides for the deployment of the calibration sources

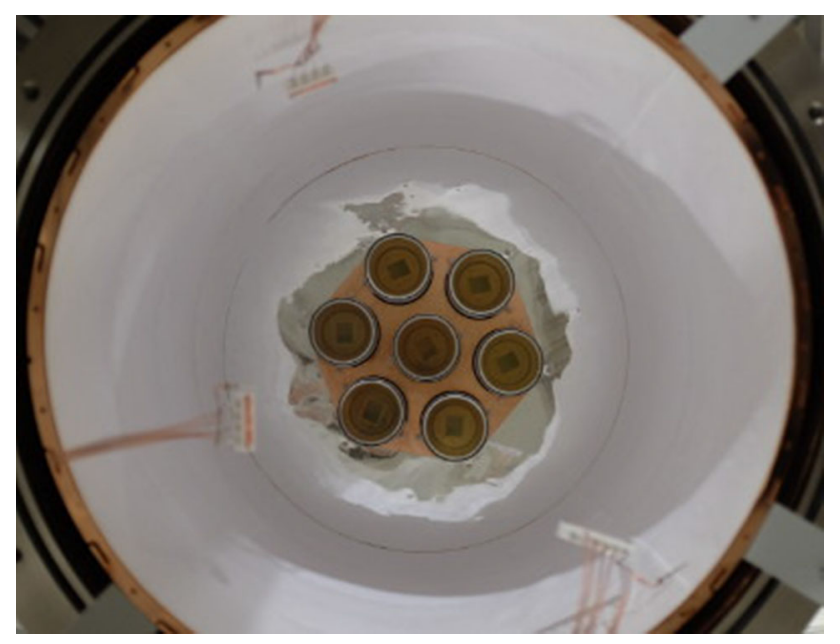

Fig. 11 Bottom PMT plate with 7 PMTs and copper cylinder. Plate and cylinder are covered by wavelength shifting Tetrate ${ }^{\circledR}$ foil soaked in TPB [24]

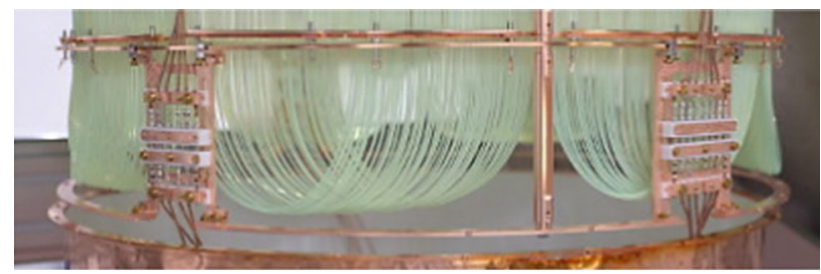

Fig. 12 PMT cable plugs custom made from copper and PTFE allow to separate the bottom LAr veto segment from the central fiber part

ous test series it was found that the first generation of R11065 PMTs was unstable under cryogenic conditions. However, in cooperation with the manufacturer it was possible to receive the current modified and improved version: each of the PMTs has been continuously operated for at least six weeks in a LAr test stand prior to the deployment in GERDA, and has been operated stably in GERDA since the start of Phase II more than one year ago (see Sect. 6).

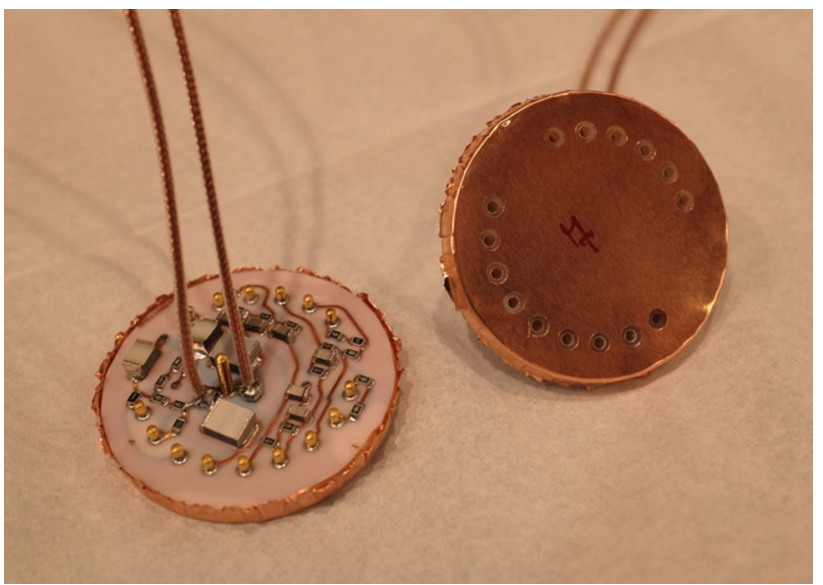

Fig. 13 Voltage divider potted into epoxy resin

The voltage dividers are designed for negative bias and high signal quality. The electrical power consumption is kept low at about $20 \mathrm{~mW}$ to prevent the argon from boiling. For further protection against discharges due to argon gas bubbles the PCB is potted into epoxy resin and a copper pot (see Fig. 13). Custom made SAMI RG178 coaxial cables are used for both signal and bias voltage inside the GERDA lock and cryostat.

The PMT signals are amplified outside of the lock by custom made shapers and split into two branches; one output leads to a FADC for digitization, and a second branch is used to independently monitor the count rates: the signals are fed into a custom made scaler device that applies a threshold of $\sim 20 \%$ photoelectron amplitude to count hits and store the rate in a database. The GERDA Slow Control [25] has access to this database and invokes an immediate automatic safety ramp down of a PMT's bias voltage in case its rate exceeds a limit of $20 \mathrm{kHz}$. The bias power supply is the same CAEN SY1527 system as used for the Cherenkov veto PMTs [5]. High voltage filters are mounted at the feedthroughs to the GERDA lock to reduce electronic pickup noise on the bias power.

The gain of the PMTs is calibrated to $(2-3) \times 10^{6}$ with bias voltages in the range from -1300 to $-1550 \mathrm{~V}$. At these settings the peak-to-valley ratio reaches its best values of 3.54 , which is relevant to identify hits above baseline noise at a low threshold. The gain is constantly monitored and found to be stable throughout the operation in GERDA (see Sect. 6).

\subsection{The fiber-SiPM system}

The middle section of the LAr veto setup (Fig. 14) consists of a curtain ( $\sim 50 \%$ coverage) of WLS fibers which are read 


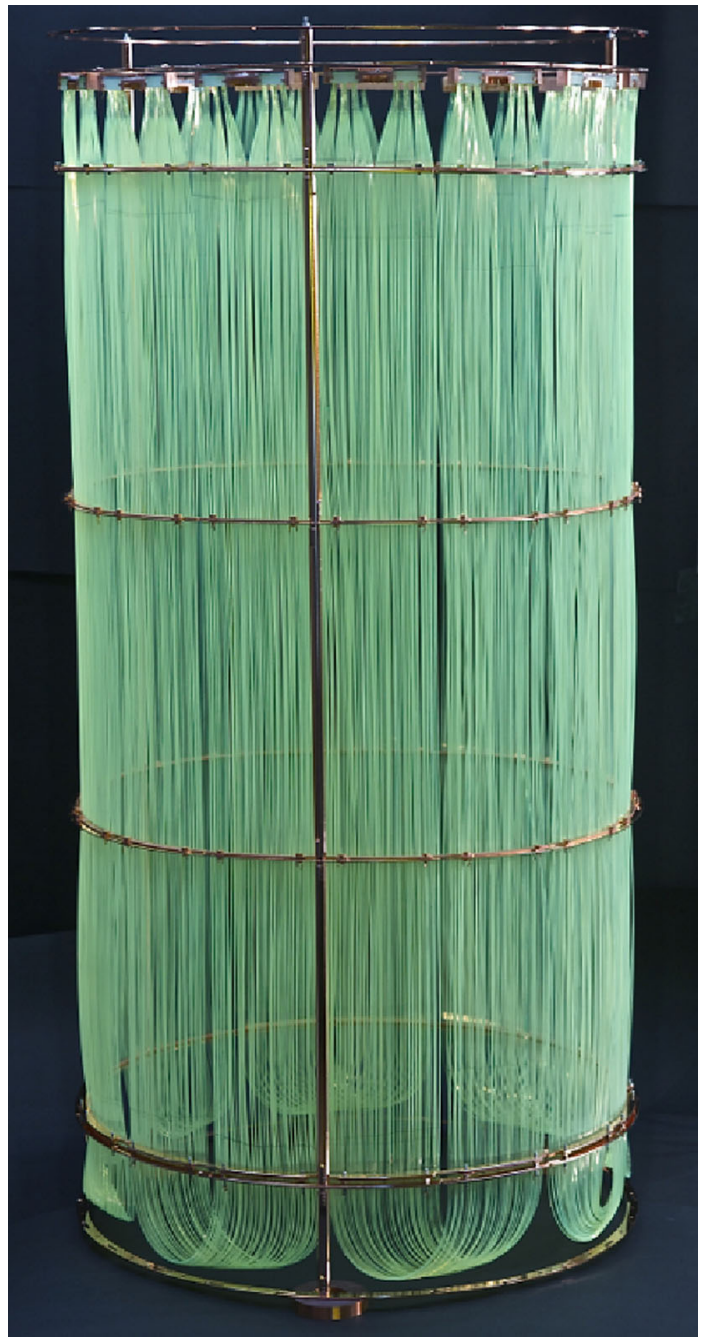

Fig. 14 The fiber curtain: height $\sim 1 \mathrm{~m}$, diameter $\sim 0.5 \mathrm{~m}$; 405 fibers read out on both ends by $90 \mathrm{SiPMs}$

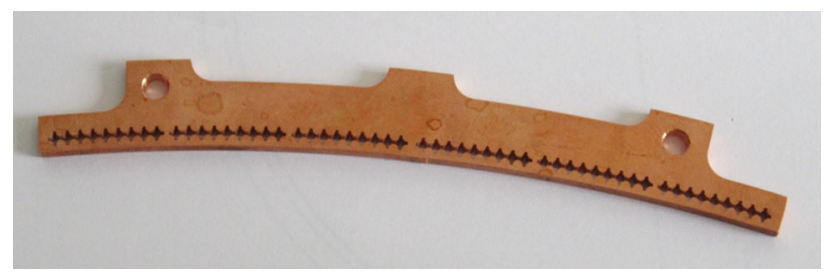

Fig. 15 Fiber holder produced with wire erosion. Note the pattern of diagonally aligned square holes. One holder covers an angular range of $23.5^{\circ}$

out with SiPMs. Note that its field of view is limited by the copper radon shroud ${ }^{3}$ at diameter $0.75 \mathrm{~m}$.

\footnotetext{
3 The radon shroud separates a central volume of about $3 \mathrm{~m}$ height and $0.75 \mathrm{~m}$ diameter from the remaining volume of the cryostat in order to prevent that radon, emanating e.g. from the walls of the vessel, may be transported by convection close to the detector array [5].
}

\subsubsection{Fiber curtain}

The design goal of the fiber detector was to achieve the largest possible coverage with light detectors while using the minimum amount of material hence minimizing the radioactivity in the vicinity of the Ge detectors.

The BCF-91A multiclad fibers from Saint-Gobain with a cross section of $1 \times 1 \mathrm{~mm}^{2}$ were chosen. The square cross section is needed for the highest possible trapping efficiency and the absorption spectrum of the BCF-91A fiber matches well the emission spectrum of the TPB. The surface of the fibers is coated with TPB by vacuum deposition.

The fibers are supported by a lightweight copper frame that also carries the weight of the bottom PMT section of the setup.

The arrangement of the square fibers is such that their diagonal is tangential to the circular flanges maximizing their surface turned towards the enclosed volume. The fibers are held in place by copper holders as the one seen in Fig. 15. These holders bundle 54 fibers in one unit which are connected to six $3 \times 3 \mathrm{~mm}^{2}$ SiPMs.

At the bottom part of the fiber shroud the fibers are bent around and fed through the neighboring copper holder (see Fig. 14) such that two pairs of copper holders form a double module. Every single fiber is about $1.8 \mathrm{~m}$ long and both of its ends are instrumented with SiPMs at the top of the cylinder.

The total amount of fibers is about $730 \mathrm{~m}$. This corresponds to a mass of about $765 \mathrm{~g}$. The total surface is about $2.9 \mathrm{~m}^{2}$ half of which is facing inwards the enclosed volume.

\subsubsection{SiPMs}

For possible use in GERDA the radioactivity of commercial SiPMs is a big concern because of the substrate that is either ceramic or ordinary glass fiber PCB material. On the other hand the purity of the silicon wafers the chips are made of is expected to be very high. To have the radiopurity issue under control it was decided to pack the SiPMs ourselves. Therefore the $3 \times 3 \mathrm{~mm}^{2}$ SiPMs were purchased in die from Ketek $\mathrm{GmbH}$.

The packaging consists of a Cuflon ${ }^{\circledR}$ PCB with square holes machined into it for the SiPM chips. Such a holder with SiPMs already implanted is shown in Fig. 16. Each holder has place for three SiPMs. The top copper layer of the PCB material is divided into two strips during the milling to form the two contacts of the SiPM array. The SiPMs are placed in the holder and bonded to the copper stripes. Then the holder is covered with a thin layer of transparent epoxy glue (Polytec EP601).

Each array was tested first at room temperature, then in liquid nitrogen $(\mathrm{LN})$. The arrays that passed the first test were assembled in double arrays of six SiPMs and tested again in LN. Only fully functional SiPM arrays with low 


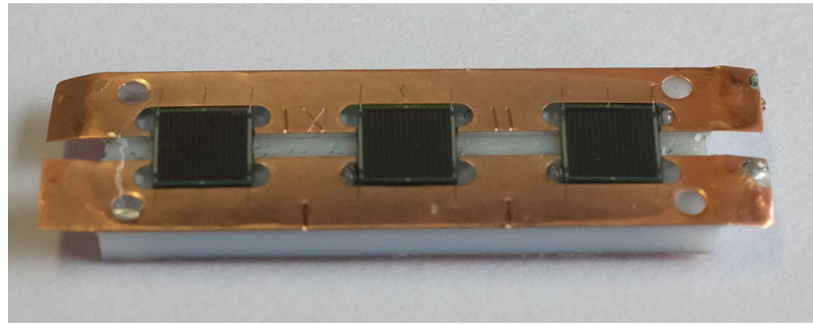

Fig. 16 Custom packaging of three $3 \times 3 \mathrm{~mm}^{2} \mathrm{SiPMs}$ on a Cuflon ${ }^{\circledR}$ holder

dark rate $\left(<1 \mathrm{~Hz} / \mathrm{mm}^{2}\right.$ at about $2.7 \mathrm{~V}$ overvoltage in $\left.\mathrm{LN}\right)$ were accepted for deployment in GERDA.

Six SiPMs are connected in parallel to one $50 \Omega$ cable in the cable chain. There is no active or passive electronic component in the LAr. The total cable length from the SiPMs to the amplifier input is about $20 \mathrm{~m}$.

The strongly temperature dependent quenching resistors of the SiPMs cause very long pixel recharge times in the range of microseconds when the SiPMs are submersed in LAr. In addition, the capacity of the large array and the cable reduces the peak amplitude of the signal significantly. The slow and small amplitude signals suggest the use of charge sensitive amplifiers.

Figure 17 shows the readout circuit schematics. Each group of three SiPMs corresponds to a unit shown in Fig. 16. The transmission line in Fig. 17 stands for the $20 \mathrm{~m}$ cable mentioned above. The potentiometer regulates the bias voltage of the SiPMs which is connected to the core of the coaxial cable. The signal is decoupled with a $100 \mathrm{nF}$ capacitor and connected to the charge sensitive amplifier (CR112 from Cremat). The bias circuit and the charge amplifier are mounted in a custom-made NIM module.

\subsection{Data acquisition and analysis}

The same FADC system (SIS 3301 Struck) used for the Ge detectors [5] records the pulse shapes of the 16 PMT and 15 SiPM channels and saves them for off-line analysis. The PMT traces are digitized with $100 \mathrm{MS} / \mathrm{s}$ and for each channel a trace of $12 \mu$ s length is saved to disk. The resolution

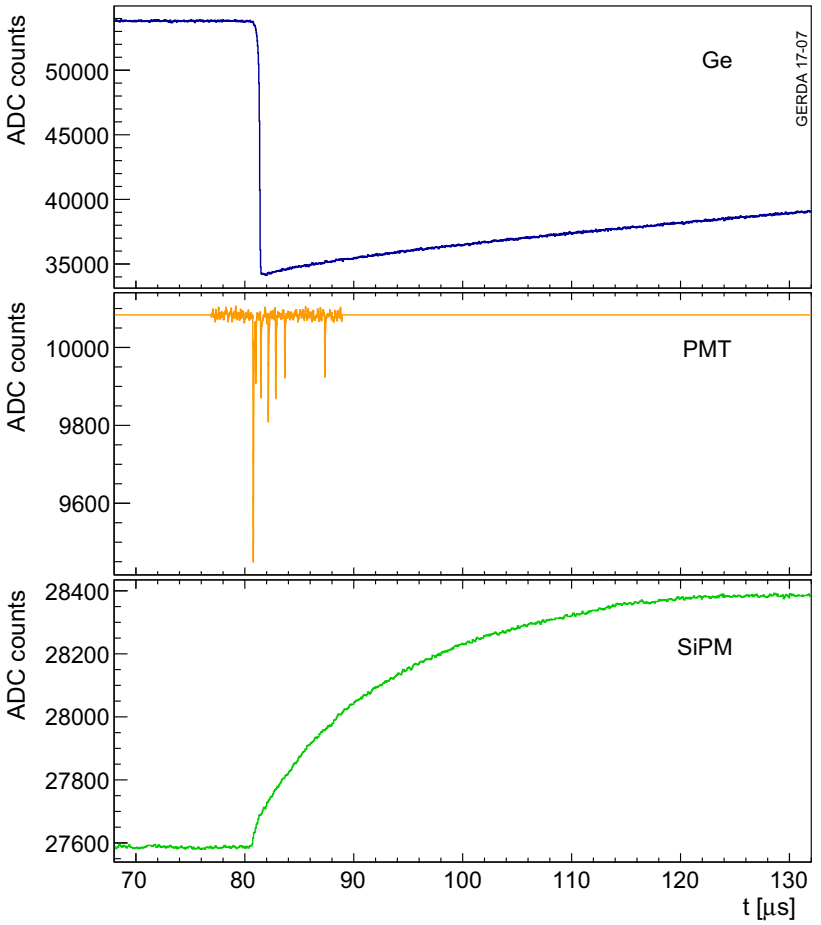

Fig. 18 Sample traces of an event with signals in a Ge detector and the LAr veto system. On top the trace of the Ge detector that triggered the event; a PMT trace in the middle - the fast component of the scintillation light is followed by several smaller pulses from the triplet component; at bottom one of the SiPMs traces with the expected slower signal (see Sect. 3.2)

of the SiPM traces is reduced to $80 \mathrm{~ns}$ to save disk space but traces of $120 \mu$ s length are recorded. All LAr channels are read out together with the Ge channels if at least one $\mathrm{Ge}$ detector has an energy deposition above $100 \mathrm{keV}$. Figure 18 shows the traces of a representative background event that has been triggered by a Ge detector and that has produced in addition signals in the PMTs and SiPMs of the LAr veto system. PMT hits are reconstructed in the offline analysis following the procedure described in [26]. First, the baseline is determined using an iterative method from [27]. Then a leading edge trigger with a threshold of 3 baseline standard deviations is applied to identify up to fifteen hits per trace. Amplitude and charge of each hit are computed. No qual-
Fig. 17 Circuit diagram of the SiPM readout. Only one channel is shown with six SiPMs in parallel which corresponds to an array of $54 \mathrm{~mm}^{2}$. The cable separating the SiPMs and the amplifier is about $20 \mathrm{~m}$ long. The charge sensitive amplifier is a Cremat-112

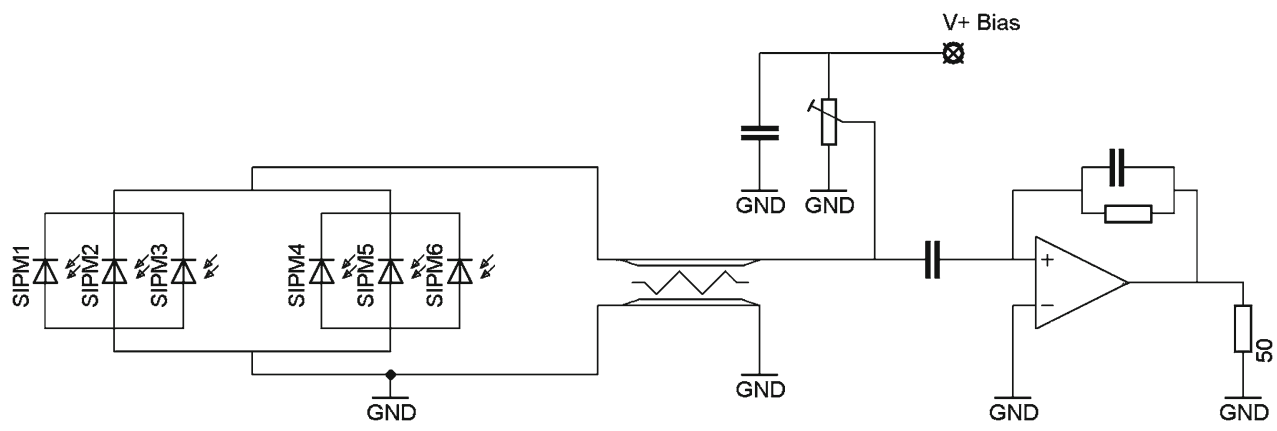


ity cuts or signal filtering is applied. The trigger positions are converted into time differences relative to the first trigger found in the Ge detector traces. Trigger positions and amplitudes are subsequently used together with hits from the SiPM to test the LAr veto condition.

SiPM hits in the recorded traces are identified with a trigger finding algorithm based on the trapezoidal filter. The moving window deconvolution is applied twice on the recorded traces. In the first step the decay time of the amplifier is deconvoluted $(50 \mu \mathrm{s})$ and in the second step the RC constant of the SiPM given by the quenching resistor times pixel capacity is removed. To determine the trigger time a trigger finding algorithm [26] is employed on the resulting waveform, and the amplitude of the pulses is read after a fixed delay following the trigger.

The algorithms were implemented in the GELATIO framework [28] which is used to process GERDA data. Each event is characterized by the calibrated energy deposited in the Ge diode, a data quality flag, the classification as signal or background event from the PSD analysis, and veto flags from the muon veto and LAr veto systems.

\section{Upgrade of infrastructure}

The break after the end of the Phase I run was used for both maintenance and upgrade work. After more than 3 years of operation the water tank was emptied. Selected welds and surfaces of both cryostat and water tank were inspected. No corrosion problems were observed, and the system safety of the pressure equipment was certified by a notified body. With the Phase I lock dismounted, a ${ }^{228}$ Th calibration source of $20 \mathrm{kBq}$ was recovered that dropped by accident during Phase I to the bottom of the cryostat. While it did not affect the Phase I background index, its presence would have not been tolerable in Phase II. For this recovery the bottom of the radon shroud had to be cut out. Both actions were performed with remotely controlled tools in the LAr-filled and hermetically closed cryostat.

\subsection{Clean room upgrade}

In order to improve the temperature stability within the clean room, the ventilation system was upgraded. An additional pump was installed that regulates the cooling water supply for the GERDA clean room ventilation system according to the needs. Flux sensors have been installed to strategic cold water tubes, allowing for real time monitoring of the cooling water throughput. These measures have led to an increase of temperature stability inside the clean room. While in Phase I the stability was about $\pm 0.7^{\circ} \mathrm{C}$ it could be now stabilized to $\pm 0.2^{\circ} \mathrm{C}$. Additional temperature sensors have been installed to the body of the lock system. These show that temperature fluctuations of the experimental volume itself are smaller than $\pm 0.1^{\circ} \mathrm{C}$. This improvement is relevant for the long term stability of the ohmic resistance of the readout cables.

\subsection{Muon veto system}

The muon veto system [29] was slightly upgraded. For the replacement of the lock the plastic muon veto system had to be removed from the roof of the clean room. After reinstallation, a broken amplifier of the plastic veto was replaced.

During the inspection of the cryostat the water tank was empty and thus a refurbishment of the PMTs of the muon veto was possible. Being accessible without scaffolding, two of four broken PMTs could be replaced by spare ones. At the beginning of Phase II, in total still 3 out of 66 Cherenkov PMTs were not working, 4 more failed during data collection. The plastic panels on the roof of the clean room (see Fig. 1) are working satisfactorily.

\subsection{Lock system}

Keeping the same functional principle, the Phase II lock replaces the Phase I twin lock system by a single-arm lock with enlarged diameter $(550 \mathrm{~mm})$ and height $(2682 \mathrm{~mm})$. This allows us to deploy both the 7 string detector array and the complete assembly of the LAr veto instrumentation (see Fig. 19). Both detector systems are suspended from a chain which allows us to lower them by $\sim 6 \mathrm{~m}$ down to the center of the cryostat. Like in Phase I, the suspension chain also serves for guiding the cables.

\subsubsection{Functionality of lock}

Two stainless steel tubes constitute the lock volume, an upper one of $1.75 \mathrm{~m}$ height, and a lower one of $0.93 \mathrm{~m}$ height. They mount vertically directly on the DN630 shutter which separates the cryostat's volume from atmosphere. Bellows and set-screws allow us to vary the length of both tubes by $\pm 5 \mathrm{~cm}$. The lock is opened by sliding the lower tube horizontally to the side after its two flanges have been detached. The resulting gap allows to introduce the three segments of the LAr veto system successively into the lock. The top PMT plate represents the interface between cable chain and the upper copper shroud; it is permanently installed in the lock resting just by its weight on a keyed index plate which is attached at the end of the cable chain. The position of the top PMT plate can be locked by three bolts at the upper end of the top tube; thus the cable chain (and the Ge detector array) can be moved independently downward. In standard operation, when only the Ge detector array has to be serviced, the top and middle segments of the LAr veto system are thus retained in the upper tube of the lock while its bottom segment is moved together with the lower tube to the side. 


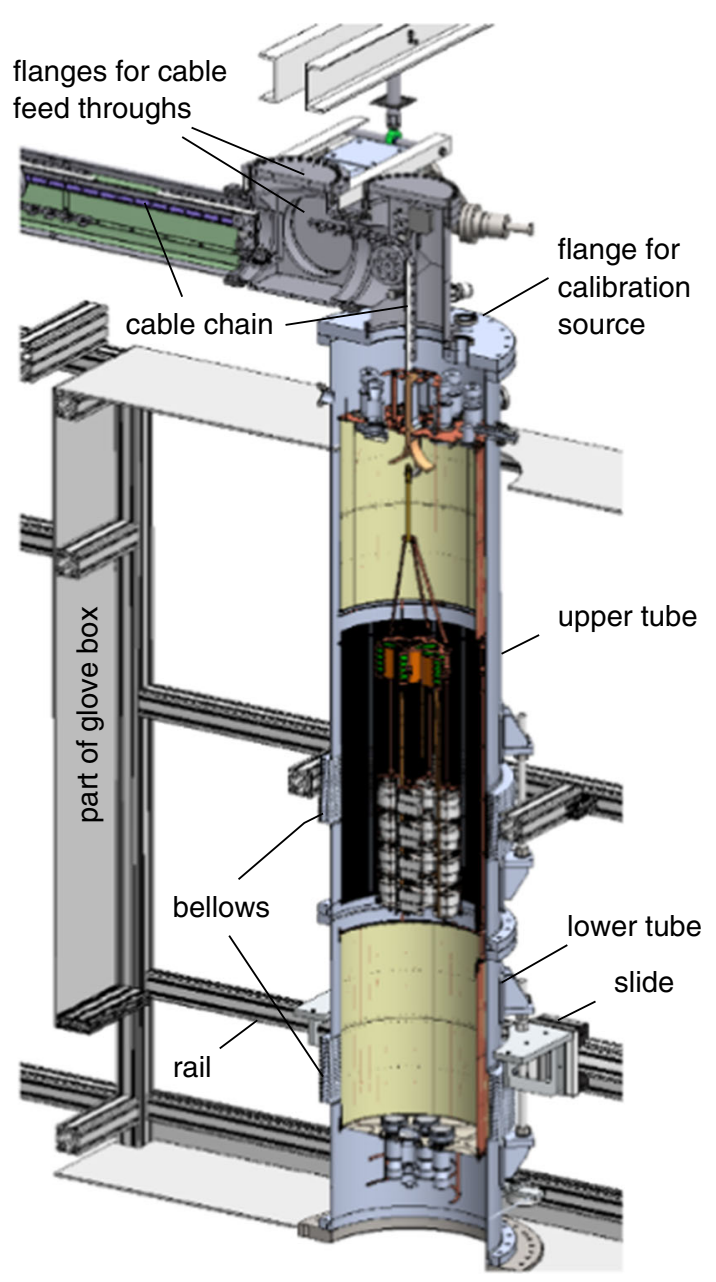

Fig. 19 Vertical part of the Phase II lock with the Ge detector array and the LAr veto system in retracted position. Both upper and lower tube are enclosed by a glove box. The lower tube can be laterally moved such that the resulting gap allows the installation and service of both the Ge detector array and the LAr veto system

All handling is done from the outside of the glove box via various appropriately positioned glove ports.

\subsubsection{Cables}

The coaxial cables deployed inside the lock are custom produced to minimize both the total radioactivity and the outgassing of nitrogen and radon in the lock and in the LAr. In fact, nitrogen impurities are powerful LAr scintillation light quenchers and $\mathrm{Rn}$ progenies can cause an increase of background. 240 pieces of $12 \mathrm{~m}$ long coaxial cables have been deployed to connect the lock feedthroughs for signal, power supplies and HV to the front end circuits, Ge detectors, and the SiPMs and PMTs of the LAr veto system. Each Ge front end circuit (see Fig. 6) requires nine coaxial cables: four for the circuit power supply, one for the pulser, and four for the amplifier outputs. The cabling serves 11 front end cir-
Table 3 List and characteristics of the coaxial cables in the 5 cable bands deployed in the GERDA Phase II cable chain

\begin{tabular}{llll}
\hline & RG178 & RG179 & $75 \Omega$ \\
\hline Purpose & LAr veto & Ge HV & Ge signal \\
Band: no of cables & V:48 & I:35 & III:61 \\
& & II:35 & IV:61 \\
AWG & 30 & 30 & 33 \\
Conductor & Cu & Cu & Cu \\
Dielectric & PFA & PFA & PFA \\
$\varnothing$ (mm) & 1.8 & 2.55 & 1.4 \\
Impedance $(\Omega)$ & 50 & 75 & 75 \\
Attenuation $(\mathrm{db} / 100 \mathrm{~m})$ & 95 & 68 & 95 \\
Capacity $(\mathrm{pF} / \mathrm{m})$ & 95 & 64 & 70 \\
Weight $(\mathrm{g} / \mathrm{m})$ & 7 & 14 & 4 \\
Resistivity $(\Omega / \mathrm{m})$ & 0.37 & 0.37 & 1.5 \\
\hline
\end{tabular}

cuits, 4 channels each plus spares. Three different cable types are deployed: RG179 for the Ge high voltage, RG178 50 $\mathrm{Ohm}$ for the SiPMs and PMTs bias and readout, and $75 \mathrm{Ohm}$ coaxial cables for the signal outputs of the Ge charge sensitive preamplifiers. Table 3 shows the list of cables and their specifications. The constituent materials have been chosen to obey cryogenics specifications and minimize space occupancy, radioactivity and outgassing rates. For the HV cables, the dielectric strength of the insulator is relevant. The perfluoroalkoxy alkane (PFA) polymer material has been chosen because of its high dielectric strength. All the cables have been custom produced by SAMI: ${ }^{4}$ both central and shielding conductor braids are in bare copper, and the dielectric and jacket material PFA has not been colored to preserve radiopurity, $\mathrm{Rn}$ emanation and minimize cable outgassing.

The cable outgassing has been measured both in vacuum tests and by chromatography on a RG179 sample of 100 $\mathrm{m}$ : thanks to high quality materials a total outgassing of $10^{-6} \mathrm{mbar} \ell / \mathrm{s}$ was achieved after $24 \mathrm{~h}$ of pumping. The measurements were performed at $40^{\circ} \mathrm{C}, 100^{\circ} \mathrm{C}$, and $150^{\circ} \mathrm{C}$. Only at $100^{\circ} \mathrm{C}$ and $150^{\circ} \mathrm{C}$, a few peaks of organic and fluor-organic compounds, octane and butane decafluoro-butane, showed up at few minutes arrival times. No peaks corresponding to known NIST Library nitrogen or nitrogen compounds were observed.

For deployment in the cable chain, the 240 cables have been woven in five cable bands of $95 \mathrm{~mm}$ width (see Table 3 ). Weaving was done with weaving machines at a commercial company. ${ }^{5}$ Special care was taken to properly clean the cables and the PTFE thread prior to weaving. All parts of the weaving machine the cables got in touch with during the weaving

\footnotetext{
${ }^{4}$ SAMI Conduttori Elettrici Speciali, Via Venezia snc, 20060 Liscate (MI)

5 PD Cable Systems, http://www.pdcablesystems.de/index.html
} 
process were disassembled and properly cleaned using ultrapure isopropanol and water. After weaving, the cable bands were once more cleaned, dried and packed under clean room conditions.

\subsubsection{Cable chain}

The cable chain and its supporting mechanics are designed to deploy a total mass of up to $60 \mathrm{~kg}$. Made from stainless steel of $1.5 \mathrm{~mm}$ thickness, its cross section $(102 \mathrm{~mm}$ width $\times$ $20 \mathrm{~mm}$ height, with a usable height of $12 \mathrm{~mm}$ ) is enlarged by a factor of 7.5 relative to Phase I in order to accommodate the cable bands for the array and LAr veto system. A $1 \mathrm{~m}$ long piece of the cable chain has been used for stress tests applying a force up to $4.95 \mathrm{kN}$, corresponding to a test load on the pulley of $\sim 200 \mathrm{~kg}$. There was no sign of critical deformation.

When retracted, the cable chain is held inside a horizontal $3.9 \mathrm{~m}$ long DN250 tube by a pulley that runs along the length of the tube guided by a linear bearing. The cable chain is deflected around this pulley by $180^{\circ}$ and above the cryostat neck by $90^{\circ}$ vertically downwards. The pulley is connected to a metal band at the far side from the cryostat neck that can be rolled up on a winch. By unrolling the metal band the pulley moves towards the cryostat neck and the array suspended to the cable chain can be lowered into the cryostat (see Fig. 10 in Ref. [5]). At the far end from the cryostat a cross is connected to the horizontal tube with two CF flanges. These contain the feedthrough for the motor axle moving the suspension system as well as the vacuum and gas ports. On the close end to the cryostat two crosses with three CF250 flanges each are connected that carry all the signal and $\mathrm{HV}$ feedthroughs including the HV filters.

For movement of the array a stepper motor EC60 with gear GP81 and encoder provided by MAXON ${ }^{6}$ is used. A magnetofluid rotational feedthrough with $20 \mathrm{~mm}$ axle provided by $\mathrm{VacSol}^{7}$ is used for transmission of the motor movement onto the winch that is moving the cable chain, hence, the Ge detector array and LAr veto system.

\subsubsection{PLC system}

A new PLC system based on a Simatic S7-300 for the control of the array movements via the stepper motor, operation of the DN630 shutter between cryostat and lock system, pumps and LED control has been built. The system has been designed to maximize safety during operation via interlocks. Two redundant induction sensors on each side of the pulley system serve as end switches allowing a calibration of the system in the up and down position. The position of the

\footnotetext{
${ }^{6}$ Maxon motor ag, http://www.maxonmotor.ch

7 VacSol GmbH, http://www.vacsol.com
}

array is obtained independently by the stepper position of the motor and a measuring tape with holes. In case a significant deviation of position measurements is determined, the PLC stops movement of the array.

\subsubsection{Fabrication and commissioning}

All parts of the internals of the lock system were specially selected and screened for radioactivity using low background HPGe detectors and/or for radon emanation prior to mounting (see Sect. 5). The tubes have been designed and tested according to the European pressure vessel code.

The horizontal part of the Phase II lock system has been fully assembled under clean room ISO 5 conditions. All parts were cleaned in an ultrasonic bath inside a wet bench using ultra-pure water and isopropanol prior to mounting. After final mounting dry tests under clean room conditions were performed to verify mechanical precision and reproducibility of the positioning of test loads. Torsion of the cable chain leads to a reproducible rotation of the load of $\sim(10 \pm 2)^{\circ}$ when fully lowered.

Once mounted at LNGS on top of the GERDA cryostat the system was tested for vacuum leaks. None could be found at the level of $10^{-8} \mathrm{mbar} \ell / \mathrm{s}$.

Before opening the DN630 shutter between lock and LAr cryostat, the system has been (and is) conditioned over $12 \mathrm{~h}$ by several pumping cycles in order to ensure a low enough outgassing rate from cables and other parts of the internal mechanical components.

\subsection{Calibration system}

The well-proven Phase I calibration system [30] has been integrated into the Phase II lock system. New sources were produced for Phase II and characterized [31]. The three individual calibration units are mounted on the top flange of the lock. Their geometrical arrangement on a mounting circle of $380 \mathrm{~mm}$ diameter and an angular distance of $120^{\circ}$ is such ("Appendix" Fig. 32) that each source, when lowered, just fits into the space between the cylinder of the LAr veto system and two neighboring outer strings of the detector array; thereby the sources enter the inner volume of the LAr veto system by three slots in the top PMT plate (see Fig. 10). A modification of the source holders, the insulation of the Ta absorber from the stainless steel band [30], prevents a previously observed HV instability in several Ge detectors.

Individual gate valves allow the units to be decoupled from the cryostat such that the respective calibration sources can be exchanged. Besides the standard ${ }^{228} \mathrm{Th}$ source of low neutron emission [31], available sources include ${ }^{226} \mathrm{Ra}$ and ${ }^{56} \mathrm{Co}$. 


\section{Material screening results}

Compared to Phase I the amount of material deployed close to the detectors has increased in Phase II, and hence the constraints for the radiopurity of the respective materials have become even more restrictive. State-of-the-art screening techniques have been used again to verify the desired radiopurity. The available screening facilities have been discussed earlier in some detail [5]. They include Ge $\gamma$ ray spectrometers in the underground laboratories at MPI-K Heidelberg, HADES (IRMM) in Belgium, and LNGS, Italy. Screening with Inductively Coupled Plasma Mass spectrometers (ICPMS) has been performed at LNGS and at INR RAS, Moscow. Radon emanation measurements were done with both the ultra-low background proportional counters developed originally at MPI-K Heidelberg for the Gallex experiment, and with the (less sensitive) permanently installed ${ }^{222} \mathrm{Rn}$ electrostatic counter at GERDA.

In the following sections the uncertainties are given as \pm 1 standard deviations and the limits are quoted at $68 \%$ CL.

\subsection{Radon emanation inside the lock}

${ }^{222} \mathrm{Rn}$ emanated inside the lock may be dissolved in the LAr and contribute to the background. Hence the selection of lowemanating construction materials is crucial. All non-metal materials mounted inside the lock have been qualified by $\mathrm{Rn}$ emanation measurements prior to assembly. Additionally, all components located close to the detector array have been screened using HPGe detectors. Like in Phase I all flanges of the lock are sealed with metal gasket against atmosphere when possible. Else Kalrez O-rings were chosen whose low emanation rate had been established in Phase I [5].

The measured Rn emanation of the woven coaxial cables bands is given in Table 4. The total emanation rate expected from all cables is lower than $4.0 \mathrm{mBq}$. This is consistent with the total ${ }^{226} \mathrm{Ra}$ activity from the woven cable bands obtained from HPGe screening (see Table 6 ) of $\sim 10 \mathrm{mBq}$, considering the fact that only a fraction of the ${ }^{222} \mathrm{Rn}$ resulting from the decays of ${ }^{226} \mathrm{Ra}$ does actually emanate from the cables.

Radon emanation measurements of the fully equipped lock system showed rates below the tolerable level. A saturation activity of $(12.5 \pm 5) \mathrm{mBq}$ was measured that has to be compared to $(55 \pm 3.5) \mathrm{mBq}$ from the cryostat alone.

Table 4 Results from Radon emanation measurements of the woven cable bands (see Table 3)

\begin{tabular}{ll}
\hline Band & Activity $(\mu \mathrm{Bq} / \mathrm{m})$ \\
\hline $48 \times \mathrm{RG178}$ & $<266$ \\
$35 \times \mathrm{RG179}$ & $<19$ \\
$61 \times 75 \Omega$ & $12 \pm 2$ \\
\hline
\end{tabular}

Table 5 Radioactive impurities of the components of one nylon minishroud (MS) from ICPMS measurements. Uncertainties are estimated to be about $30 \%$

\begin{tabular}{lllll}
\hline Component & $\mathrm{U}(\mathrm{ppt})$ & $\mathrm{Th}(\mathrm{ppt})$ & $\mathrm{K}(\mathrm{ppb})$ & Mass $(\mathrm{g})$ \\
\hline TPB & 10 & 9 & 65 & \\
Polystyrene & $<5$ & 10 & 100 & \\
Glue & $<10$ & $<10$ & 900 & \\
Nylon & $<10$ & $<15$ & - & 27.6 \\
Nylon coated & 11 & 18 & $<25$ & \\
Nylon glued & 38 & 39 & 1200 & \\
\hline MS finished & $6.1 \mu \mathrm{Bq}$ & $2.6 \mu \mathrm{Bq}$ & $242 \mu \mathrm{Bq}$ & 28.1 \\
\hline
\end{tabular}

\subsection{Nylon mini-shrouds}

The radioactive purity of the coated nylon films was checked by ICPMS measurements at LNGS [32]. Results of the measurements are shown in Table 5. Differences in radiopurity of similar samples indicated that surface contaminations play a big role; so it is important to prepare and keep foils in a clean condition. Coating of the nylon foils was performed in a clean room by brushing: this allows to deposit a small amount of WLS with a good enough coverage. The deposited WLS was determined by weighing the nylon before and after coating. A typical mass of the coating was about $0.3 \mathrm{mg} / \mathrm{cm}^{2}$ for the nylon film coated from both sides.

To assess if the radiopurity level is acceptable, a detailed simulation of the nylon MS in the GERDAPhase II setup was performed [33]. The expected contribution of all minishrouds to the background index is about $5 \times 10^{-4}$ $\mathrm{cts} /(\mathrm{keV} \mathrm{kg}$ year) before LAr veto and PSD cuts, and two orders of magnitude lower after these cuts.

\subsection{Coaxial cables}

Table 6 compares the length-specific activities of the coaxial cables deployed in the lock in Phase I and II. In Phase II, the high voltage cables (RG179) are factors of about 15 to 25 better in ${ }^{226} \mathrm{Ra}$ and ${ }^{228} \mathrm{Th}$, and a factor of 50 better in ${ }^{40} \mathrm{~K}$ than in Phase I (TR11.18kV); compared to the standard production SAMI cables, they are better by factors of about 20 to 30 in ${ }^{226} \mathrm{Ra}$ and ${ }^{228} \mathrm{Th}$ and ${ }^{40} \mathrm{~K}$. The ${ }^{108 m} \mathrm{Ag}$ and ${ }^{110 m} \mathrm{Ag}$ isotopes are not present in the Phase II cables due to the bare copper choice for the conductor.

\subsection{Electronic front-end}

Table 7 reports the measured activities of the FE devices. Compared to Phase I, the new FE circuits and contacts exhibit a significantly lower contamination, which is reduced for ${ }^{226} \mathrm{Ra}$ and ${ }^{228} \mathrm{Th}$ by a factor of 1.5 and 30 per channel, respectively. For a BI of $10^{-3} \mathrm{cts} /(\mathrm{keV} \mathrm{kg}$ year) before $\mathrm{LAr}$ veto and 
Table 6 The length-specific activities of the custom coaxial cables deployed in GERDA Phase II compared to the standard cables used in Phase I. All screening has been done with Ge $\gamma$ spectrometers. Phase II cables were woven in bands (see Table 3)

\begin{tabular}{|c|c|c|c|c|c|c|c|}
\hline Cable & ${ }^{226} \mathrm{Ra}(\mu \mathrm{Bq} / \mathrm{m})$ & ${ }^{228} \mathrm{Th}(\mu \mathrm{Bq} / \mathrm{m})$ & ${ }^{40} \mathrm{~K}(\mu \mathrm{Bq} / \mathrm{m})$ & ${ }^{60} \mathrm{Co}(\mu \mathrm{Bq} / \mathrm{m})$ & ${ }^{137} \mathrm{Cs}(\mu \mathrm{Bq} / \mathrm{m})$ & ${ }^{108 m} \mathrm{Ag}(\mu \mathrm{Bq} / \mathrm{m})$ & ${ }^{110 m} \mathrm{Ag}(\mu \mathrm{Bq} / \mathrm{m})$ \\
\hline \multicolumn{8}{|l|}{ Phase I } \\
\hline RG178 & $11 \pm 3$ & $13 \pm 5$ & 680 & $<0.4$ & - & $13 \pm 2$ & $8 \pm 1$ \\
\hline TR11.18kV & 134 & 96 & 3700 & - & n.a. & n.a. & n.a. \\
\hline HABIA50 & $<5$ & $3 \pm 2$ & 1200 & $<1$ & - & $2 \pm 1$ & $4 \pm 2$ \\
\hline \multicolumn{8}{|l|}{ Phase II } \\
\hline 75 Ohm & $5 \pm 1$ & $5 \pm 1$ & $190 \pm 30$ & $<1$ & $3 \pm 1$ & - & - \\
\hline RG178 & $4 \pm 1$ & $<5$ & $81 \pm 16$ & $<2$ & - & - & - \\
\hline RG179 & $<5$ & $6 \pm 2$ & $74 \pm 15$ & $<2$ & - & - & - \\
\hline
\end{tabular}

Table 7 The activity of the GERDA front-end cables and PCBs in Phase I and II. The Phase II front-end PCBs have been screened fully populated while the Phase I samples had no contact pins yet. The values of the
Phase I readout/HV 'cable', a high-purity copper wire within a PTFE tube, refer to its insulation

Mass $(\mathrm{g}) \quad{ }^{226} \mathrm{Ra}(\mu \mathrm{Bq} /$ piece $) \quad{ }^{228} \mathrm{Th}(\mu \mathrm{Bq} /$ piece $) \quad{ }^{40} \mathrm{~K}(\mu \mathrm{Bq} /$ piece $) \quad{ }^{60} \mathrm{Co}(\mu \mathrm{Bq} /$ piece $) \quad{ }^{137} \mathrm{Cs}(\mu \mathrm{Bq} /$ piece $)$

Phase I

\begin{tabular}{|c|c|c|c|c|c|c|}
\hline readout/HV 'cable' (1 m) & 4.28 & $4.7 \pm 0.9$ & $<3.3$ & $34 \pm 9$ & - & $<1.5$ \\
\hline 3-ch front end PCB w/o pins & 5.2 & $290 \pm 100$ & $140 \pm 60$ & $1900 \pm 700$ & $<56$ & $<42$ \\
\hline \multicolumn{7}{|l|}{ Phase II } \\
\hline Pyralux ${ }^{\circledR} 3$ mil $(80 \mathrm{~cm})$ & 0.5 & $<1.9$ & $<2.3$ & $55 \pm 15$ & $<0.4$ & $<0.6$ \\
\hline Cuflon $^{\circledR} 2 \operatorname{mil}(90 \mathrm{~cm})$ & 0.5 & $27 \pm 6$ & $<13$ & $120 \pm 60$ & $<9.2$ & $<5.5$ \\
\hline Cuflon ${ }^{\circledR} 10 \mathrm{mil}(90 \mathrm{~cm})$ & 2.7 & $21 \pm 6$ & $<15$ & $300 \pm 60$ & $6 \pm 3$ & $<6.7$ \\
\hline 4-ch front end PCB & 17 & $240 \pm 40$ & $<120$ & $1200 \pm 350$ & $<28$ & $<69$ \\
\hline
\end{tabular}

PSD cuts, Monte Carlo (MC) simulations predict maximum allowed activities of $2 \mathrm{mBq}$ and $0.5 \mathrm{mBq}$, respectively, if the electronics boards are mounted $30 \mathrm{~cm}$ above the top detectors. The total activity of the 10 boards amounts to $2.3(3) \mathrm{mBq}$ and $0.7(4) \mathrm{mBq}$ for ${ }^{226} \mathrm{Ra}$ and ${ }^{228} \mathrm{Th}$, respectively, being thus close to the radiopurity limit.

For the front-end cables the corresponding limits are $0.5 \mu \mathrm{Bq} / \mathrm{cm}$ for ${ }^{226} \mathrm{Ra}$ and $0.04 \mu \mathrm{Bq} / \mathrm{cm}$ for ${ }^{228} \mathrm{Th}$. The activity of the Pyralux ${ }^{\circledR}$ cables is well below the limit for ${ }^{226} \mathrm{Ra}$ and almost meets the limit for ${ }^{228} \mathrm{Th}$. The Cuflon ${ }^{\circledR}$ cables just meet the limit for ${ }^{226} \mathrm{Ra}$ and miss it for ${ }^{228} \mathrm{Th}$ by about a factor of 10 . With $60 \%$ of the deployed front-end cables being of the Cuflon ${ }^{\circledR}$ type this excess reduces to a factor of $\sim 4$; this is still tolerable since the LAr veto alone suppresses the ${ }^{228}$ Th induced background by about a factor of 100 (see Sect. 6).

\subsection{Detector holders}

Table 8 compares the radiopurity of Phase I and Phase II detector holders. The substitution of copper by silicon mounting material results in significantly reduced ${ }^{228} \mathrm{Th}$ and ${ }^{226} \mathrm{Ra}$ activities. The corresponding contribution to the BI is less than $10^{-5} \mathrm{cts} /(\mathrm{keV} \mathrm{kg}$ year) according to simulations before LAr veto and PSD cuts.

\subsection{LAr veto system}

The radiopurity of the components of the LAr veto system is critical since they add a large amount of new material close to the detector array. The activities of the components as well as the estimated background contributions of the PMT and fiber systems are given in Tables 9 and 10, respectively.

The specification was that both systems contribute about equally to the BI. The sixteen 3" PMTs and voltage dividers exhibit the largest specific activities although the radiopurity of the PMTs had been further improved in collaboration with the manufacturer, and the potted voltage dividers have been fabricated from selected materials, $0.5 \mathrm{~mm} \mathrm{Cuflon}{ }^{\circledR}$ as printed circuit board and film capacitors rather than ceramic ones. Nevertheless, the PMTs with their voltage dividers had to be mounted at a minimum distance of more than $1 \mathrm{~m}$ from the closest Ge detector in order to stay within the background budget. On the other hand, the very low specific activity of the fibers allows to deploy them, almost $1 \mathrm{~kg}$ of material, rather close to the detector array. Altogether, both subdetectors contribute about $1.2 \times 10^{-3} \mathrm{cts} /(\mathrm{keV} \mathrm{kg}$ year) to the 
Table 8 Comparison of the radiopurity of the Phase I and II detector holders (for masses of construction materials see Table 2). Specific activities from neutron activation analysis (NAA) [34] and ICPMS assume secular equilibrium. The background index at $Q_{\beta \beta}$ is estimated after anti-coincidences but before LAr veto and PSD

\begin{tabular}{|c|c|c|c|c|c|c|c|c|c|}
\hline \multirow[t]{2}{*}{ Material } & \multirow[t]{2}{*}{ Method } & \multicolumn{3}{|l|}{ Specific activity } & \multicolumn{3}{|l|}{ Total activity } & \multicolumn{2}{|c|}{ Background index } \\
\hline & & ${ }^{228} \mathrm{Th}(\mu \mathrm{Bq} / \mathrm{kg})$ & ${ }^{226} \mathrm{Ra}(\mu \mathrm{Bq} / \mathrm{kg})$ & ${ }^{40} \mathrm{~K}(\mu \mathrm{Bq} / \mathrm{kg})$ & ${ }^{228} \mathrm{Th}(\mu \mathrm{Bq})$ & ${ }^{226} \mathrm{Ra}(\mu \mathrm{Bq})$ & ${ }^{40} \mathrm{~K}(\mu \mathrm{Bq})$ & $\begin{array}{l}{ }^{208} \mathrm{Tl}\left(10^{-6} \mathrm{cts} /\right. \\
\text { (keV kg year) }\end{array}$ & $\begin{array}{l}{ }^{214} \mathrm{Bi}\left(10^{-6} \mathrm{cts} /\right. \\
\text { (keV kg year) }\end{array}$ \\
\hline \multicolumn{10}{|l|}{ Phase I } \\
\hline $\mathrm{Cu}$ & $\gamma$ spec. & $<19$ & $<15$ & - & $<1.6$ & $<1.3$ & - & $<15$ & $<6.6$ \\
\hline \multirow[t]{2}{*}{$\mathrm{Si}$} & NAA & $<10^{-3}$ & $<10^{-4}$ & - & - & - & - & - & - \\
\hline & $\gamma$ spec. & - & - & $4.3(9) \times 10^{3}$ & - & - & $4.3(9)$ & - & - \\
\hline PTFE & $\gamma$ spec. & $30(14)$ & $25(9)$ & 600 & $0.21(10)$ & $0.18(6)$ & 4.2 & $1.9(9)$ & $0.9(3)$ \\
\hline \multicolumn{10}{|l|}{ Phase II } \\
\hline $\mathrm{Cu}$ & $\gamma$ spec. & $<19$ & $<15$ & - & $<0.25$ & $<0.20$ & - & $<3.7$ & $<1.6$ \\
\hline \multirow[t]{2}{*}{$\mathrm{Si}$} & NAA & $<10^{-3}$ & $<10^{-4}$ & - & - & - & - & - & - \\
\hline & $\gamma$ spec. & - & - & $4.3(9) \times 10^{3}$ & - & - & $86(18)$ & - & - \\
\hline PTFE & $\gamma$ spec. & $67(25)$ & $50(20)$ & $<240$ & $0.07(3)$ & $0.05(2)$ & $<0.24$ & $1.1(4)$ & $0.4(2)$ \\
\hline CuSn6 & ICPMS & $<300$ & $<300$ & - & $<0.2$ & $<0.2$ & - & $<2.8$ & $<1.6$ \\
\hline
\end{tabular}

Table 9 Activity of the components of the PMT LAr veto system and their estimated background contribution BI at $Q_{\beta \beta}$ after anti-coincidences but before the LAr veto and PSD. (pc: piece)

\begin{tabular}{|c|c|c|c|c|c|}
\hline Component & ${ }^{228} \mathrm{Th}(\mu \mathrm{Bq})$ & ${ }^{226} \mathrm{Ra}(\mu \mathrm{Bq})$ & ${ }^{40} \mathrm{~K}(\mu \mathrm{Bq})$ & $\begin{array}{l}\mathrm{BI}\left({ }^{208} \mathrm{Tl}\right)\left(10^{-6} \mathrm{cts} /\right. \\
(\mathrm{keV} \text { kg year }))\end{array}$ & $\begin{array}{l}\mathrm{BI}\left({ }^{214} \mathrm{Bi}\right)\left(10^{-6} \mathrm{cts} /\right. \\
(\mathrm{keV} k g \text { year }))\end{array}$ \\
\hline PMTs & $<1940 / \mathrm{pc}$ & $<1700 / \mathrm{pc}$ & $<9100 / \mathrm{pc}$ & $<245$ & $<33$ \\
\hline Voltage dividers & $<500 / \mathrm{pc}$ & $<1140 / \mathrm{pc}$ & $<11500 / \mathrm{pc}$ & $<63$ & $<22$ \\
\hline SAMI RG178 cables & $<14.4 / \mathrm{m}$ & $<11.2 / \mathrm{m}$ & $81(16) / \mathrm{m}$ & & \\
\hline Along $\mathrm{Cu}$ shrouds & & & & $<16$ & $<2$ \\
\hline Along Fiber shroud & & & & $<227$ & $<37$ \\
\hline Copper shrouds & $37 / \mathrm{kg}$ & $148 / \mathrm{kg}$ & - & $8.6(1)$ & $5.7(1)$ \\
\hline Tetratex ${ }^{\circledR}$ coated & $70 / \mathrm{m}^{2}$ & $150 / \mathrm{m}^{2}$ & $9800 / \mathrm{m}^{2}$ & $18.2(2)$ & $6.4(1)$ \\
\hline
\end{tabular}

Table 10 Activity of the components of the fiber-SiPM LAr veto system and their estimated background contribution BI at $Q_{\beta \beta}$ after anticoincidences but before the LAr veto and PSD

\begin{tabular}{llllllll}
\hline Component & Method & ${ }^{228} \mathrm{Th}(\mathrm{mBq} / \mathrm{kg})$ & ${ }^{226} \mathrm{Ra}(\mathrm{mBq} / \mathrm{kg})$ & ${ }^{40} \mathrm{~K}(\mathrm{mBq} / \mathrm{kg})$ & mass $(\mathrm{g})$ & $\begin{array}{l}\mathrm{BI}\left({ }^{208} \mathrm{Tl}\right)\left(10^{-6} \mathrm{cts} /\right. \\
(\mathrm{keV} \mathrm{kg} \mathrm{year}))\end{array}$ & $\begin{array}{l}\mathrm{BI}\left({ }^{214} \mathrm{Bi}\right)\left(10^{-6} \mathrm{cts} /\right. \\
(\mathrm{keV} \mathrm{kg} \text { year}))\end{array}$ \\
\hline Fiber BCF-91A & ICPMS & 0.058 & 0.042 & 0.46 & 765 & 238 & $175(1)$ \\
Plastic opt.coupl. & $\gamma$ spec. & $0.15(8)$ & $<0.19$ & 3.0 & 32 & $7(3)$ & $<0.64$ \\
SiPM & ICPMS & $<1$ & $<3$ & - & 1.3 & $<1.8$ & $<0.41$ \\
Cuflon ${ }^{\circledR}$ & $\gamma$ spec. & $0.8(5)$ & $1.3(4)$ & 18 & 15 & $16(10)$ & $2.1(6)$ \\
Pins & $\gamma$ spec. & $<5.8$ & $15(3)$ & 220 & 9.4 & $<74$ & $15(3)$ \\
Screws & $\gamma$ spec. & $<5.8$ & $15(4)$ & $<120$ & 12.7 & $<100$ & $20(5)$ \\
Glue EP601 & $\gamma$ spec. & $<1.5$ & $<0.55$ & $<13$ & 4.0 & $<8.1$ & $<0.23$ \\
\hline
\end{tabular}

BI at $Q_{\beta \beta}$ before LAr veto and PSD; that is more than the Phase II goal. As shown in Sect. 6 the LAr veto alone suppresses, however, the backgrounds at $Q_{\beta \beta}$ from ${ }^{228} \mathrm{Th}$ and ${ }^{226} \mathrm{Ra}$ sources by factors of about 100 and 6, respectively, resulting in BI values that are fully acceptable for Phase II.
Preliminary MC simulations with photon tracking predict indeed an even larger suppression yielding a total contribution of the LAr veto hardware to the BI at $Q_{\beta \beta}$ of about $10^{-5} \mathrm{cts} /(\mathrm{keV} \mathrm{kg}$ year) after LAr veto and PSD cuts. 


\section{Performance}

Phase II physics data taking started on December 25, 2015. A first data release PIIa has been presented after about 6 months of running at the Neutrino Conference 2016, and the results have been published in Nature [3]. The following discussion considers the Phase II data taking period up to April 15, 2017. About $6.7 \%$ of the total of 477 calendar days were devoted to calibration or maintenance, the rest to physics data taking. A series of nearby earthquakes with magnitudes up to 5.5 slightly affected the ongoing measurements. The exposure taken on tape with the enriched detectors amounts to $43.4 \mathrm{~kg}$ year which corresponds to a duty factor of more than $93 \%$. Data validation reduces this exposure by $9 \%$ to $34.4 \mathrm{~kg}$ year. As of April 15 2017, all deployed detectors (see "Appendix" Fig. 33) are operational except the enriched detector GD91C which has a damaged JFET. The two gaps in Fig. 21 refer to GD02D and GD91B (see Fig. 33) which are not included in our analysis since they do not exhibit full charge collection and are used thus in anti-coincidence mode only.

The performance of the detector system is characterized in particular by the stability of its energy calibration as well as by the resolutions of the various detector components. It is assessed by regular calibrations and by monitoring continuously the flow of physics data and test pulser events. The following sections present representative examples for the various components and an evaluation of the full system performance including the achieved level of background suppression.

\subsection{Ge detectors}

The operation of bare Ge crystals in LAr is a non-standard technique, and the stability of leakage currents was a major issue in the preparations for Phase I [5]. It was found that passivated detectors - those with a passivation layer in the insulating groove between $\mathrm{p}^{+}$contact and the conductive lithium $\mathrm{n}^{+}$layer - showed constantly increasing leakage current from repeated calibrations, presumably due to the build up of charges in the groove. Non-passivated detectors did not show this effect, and hence all semi-coaxial detectors deployed in Phase I had the passivation layer removed. To speed up the refurbishment and delivery of the Phase II detectors, the removal of the standard passivation was given up for quite a number of detectors (see yellow colored detectors in “Appendix" Fig. 33).

Figures 20 and 34 of the Appendix show the evolution of the leakage current for all Phase II Ge detectors in the time span between December 2015 and April 2017. In the first months, a temporary increase of leakage currents during calibration has been observed for several detectors, passivated and non-passivated ones. This is visible by current

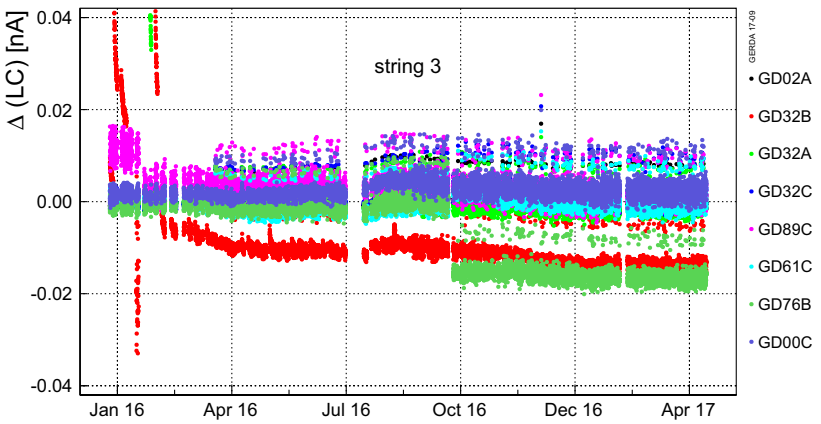

Fig. 20 Changes of leakage currents $\Delta(\mathrm{LC})$ of indicated detectors of string 3 in the period from December 2015 to April 2017. For the other detector strings the corresponding data are shown in Fig. 34 of the "Appendix"

spikes reaching up to $300 \mathrm{pA}$ immediately after irradiation; they return to the previous current values within half a day. Except for one detector, the leakage currents of all detectors, both passivated and non-passivated ones, are stable since May 2016 or even reduced compared to the beginning. Hence the number of stable detectors included in the analysis has increased in time. Thus, our experience is strikingly contrary to a former report about 'the limited long-term stability of naked detectors in $\mathrm{LN}$ as result of increasing leakage current' [35].

\subsubsection{Energy scale stability and resolution}

Energy calibrations with a ${ }^{228} \mathrm{Th}$ source are performed regularly, typically once per week; a calibration spectrum is shown in Sect. 6.2 (Fig. 27). The energy reconstruction is performed in the off-line analysis of the digitized charge pulses using either a standard semi-Gaussian shaping or, for improved energy resolution, a finite-length zero-area cusp filter [36]. The position of the various peaks are used to establish the energy scale (gain) as well as the resolution as a function of energy. The ${ }^{208} \mathrm{Tl} \gamma$ line at $2614.5 \mathrm{keV}$ provides a convenient measure to monitor the resolution close to the $Q_{\beta \beta}$ energy. The upper part of Fig. 21 shows for all detectors the measured FWHM resolutions for this line as well as for its double escape peak (DEP) at $1592.5 \mathrm{keV}$. The coaxial detectors are in strings 2, 5, and 7. Most of them have a resolution of less than $4.0 \mathrm{keV}$ FWHM. In the period from December 2015 to April 2017 the interpolated average energy resolution at $Q_{\beta \beta}$ is 3.90(7) $\mathrm{keV}$ FWHM, i.e. better than in Phase I (4.8(2) keV). The resolutions of the BEGe detectors show unexpectedly strong variations between 2.5 and $5.6 \mathrm{keV}$. At $Q_{\beta \beta}$ the interpolated mean is $2.93(6) \mathrm{keV}$ which is also better than in Phase I (3.2(2) keV). The top detector in each string exhibits indeed the expected resolution well below $3 \mathrm{keV}$ but there is a consistent trend of decreasing resolution when going down in the string. This effect 


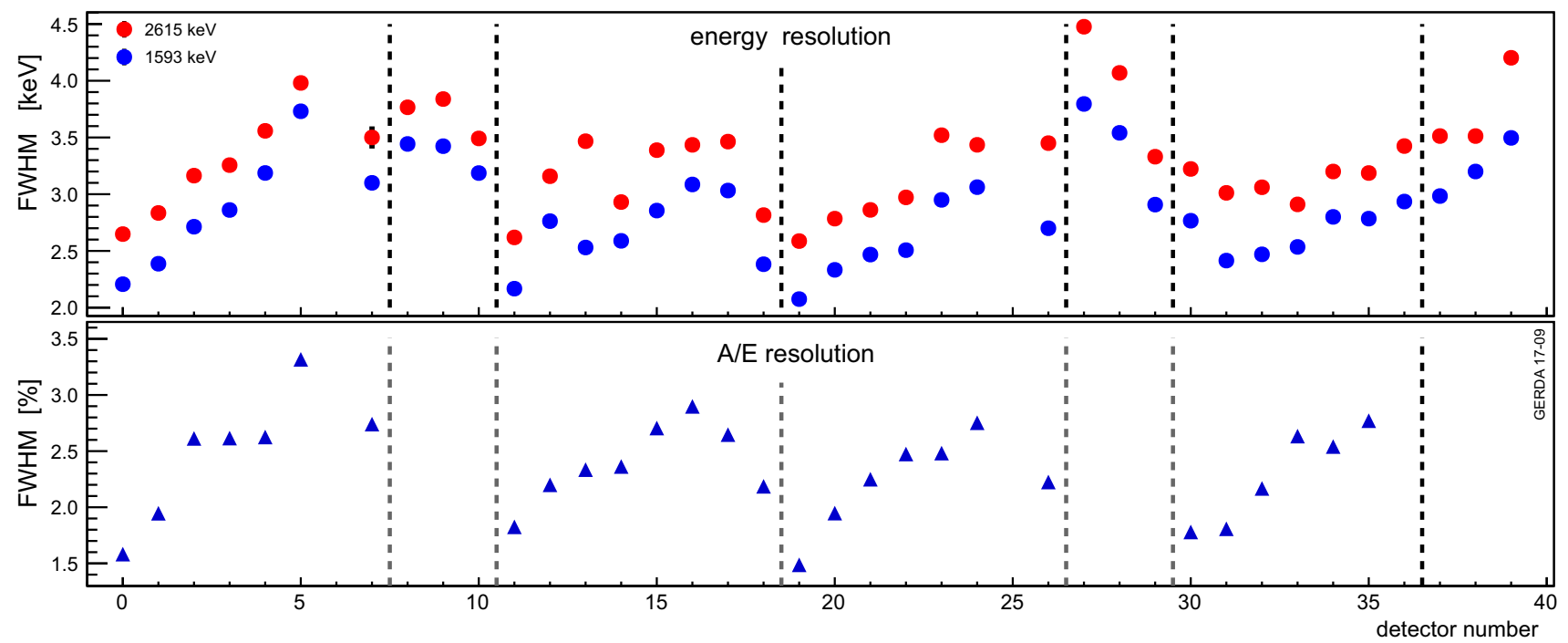

Fig. 21 FWHM energy resolutions (top) of the $2615 \mathrm{keV}$ line and its DEP at $1593 \mathrm{keV}$, and A/E resolutions (bottom) of individual Ge detectors deduced from the DEP [37]. The dashed lines separate the 7 detector strings; within each string the detector number increases from top to bottom detector

has not been observed in Phase I where single wires were used for the signal readout instead of the FFCs in Phase II. Thus larger stray capacitances with increasing cable length and capacitive coupling within the readout cable bundle of a string might explain the observation. A good understanding of the resolution pattern is, however, still lacking and further work on this issue is in progress.

The stability of the energy scale is not only verified by calibration measurements but continuously monitored by injecting every $20 \mathrm{~s}$ a test charge into the electronic front ends. If the gain variation is larger than $0.1 \%$, an extra unscheduled calibration with the ${ }^{228} \mathrm{Th}$ source is taken. Figure 22 shows for the period from December 2015 to April 2017 the shifts of the $2615 \mathrm{keV}$ calibration peak between successive calibrations. Most data points are within the range of $\pm 1 \sigma \approx \pm 1.5 \mathrm{keV}$; this is sufficient to allow the merging of the data from all periods (periods with shifts exceeding $2 \mathrm{keV}$ are discarded from analysis).

\subsubsection{Pulse shape discrimination efficiencies}

The new Ge detectors for Phase II have been chosen to be of the BEGe type because the time profile of its current pulse allows for a powerful but simple discrimination between $0 \nu \beta \beta$-like events, i.e. localized single-site events (SSEs), from background events which have often multiple energy depositions (multi-site events, MSEs) or occur at the detector surface [13]. It is based on a single parameter, the ratio $\mathrm{A} / \mathrm{E}$ of the maximum of the current pulse A over the total energy E. SSEs are identified by an (normalized) A/E value of about 1 , MSEs and events on the $\mathrm{n}^{+}$surface by a value lower than 1 , and surface events at the $\mathrm{p}^{+}$contact like $\alpha$ particles
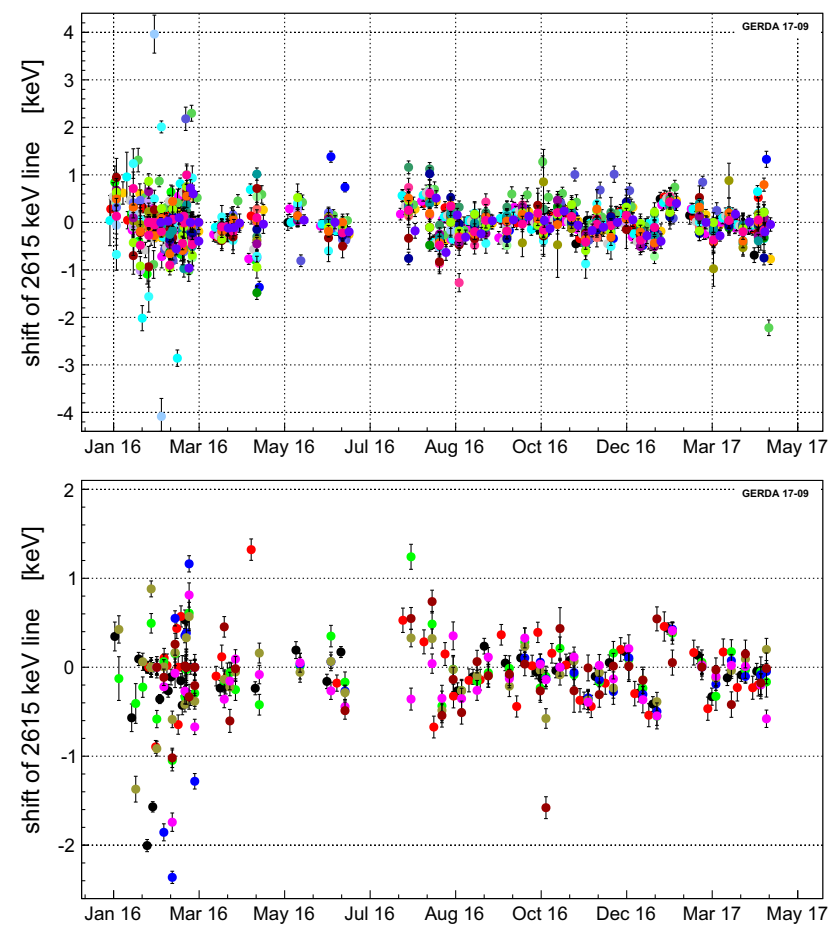

Fig. 22 Shift of the $2615 \mathrm{keV}$ peak of ${ }^{208} \mathrm{Tl}$ between consecutive calibrations for indicated BEGe (top) and enriched coaxial detectors (bottom)

by $\mathrm{A} / \mathrm{E}>1$. Like in Phase I the A/E cut is calibrated with the data from the weekly calibrations with the ${ }^{228} \mathrm{Th}$ source. Events in the DEP are used as a proxy for $0 \nu \beta \beta$ events. The inset in ${ }^{228}$ Th calibration spectrum (Fig. 27) illustrates the effect of the $\mathrm{A} / \mathrm{E} \sim 1$ cut (yellow colored spectrum): the obvious survival of the DEP at $1593 \mathrm{keV}$ and the distinct suppression of the single gamma line of ${ }^{212} \mathrm{Bi}$ at $1621 \mathrm{keV}$. 
Fig. 23 Survival efficiencies for the double escape (DEP) and single escape peak (SEP) of the $2615 \mathrm{keV} \gamma$ line of ${ }^{208} \mathrm{Tl}$, for the full energy $\gamma$ peaks (FEPs) at $1620 \mathrm{keV}$ and $2615 \mathrm{keV}$, and the Compton events around $\mathrm{Q}_{\beta \beta}$ [37]. Uncertainties are partly smaller than the symbols

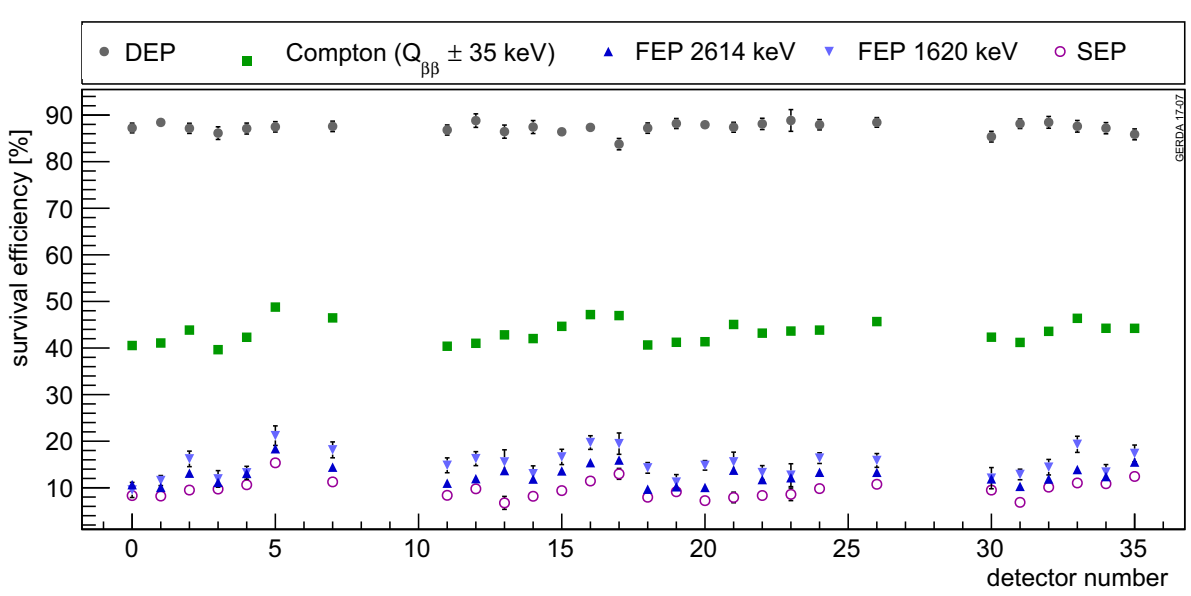

While a detailed account of the PSD analysis of Phase II data is in preparation we discuss here just some differences compared to the analysis of the Phase I data. To select signallike events a two-sided A/E cut is applied: MSE and $\mathrm{n}^{+}$surface events are removed by a cut on the low $\mathrm{A} / \mathrm{E}$ side, while events on the $\mathrm{p}^{+}$contact are rejected by a high $\mathrm{A} / \mathrm{E}$ cut. The lower part of Fig. 21 shows the strong variation of the $\mathrm{A} / \mathrm{E}$ resolution of individual BEGe detectors following a similar trend as the energy resolution. The A/E resolutions differ for individual detectors by up to a factor of 2 , and they are often significantly worse than in Phase I where they varied between 1.5 and $1.9 \%$. Moreover, to reach the Phase II BI goal of $10^{-3} \mathrm{cts} /(\mathrm{keV} \mathrm{kg}$ year), a stronger low $\mathrm{A} / \mathrm{E}$ cut for higher suppression of MSE compared to Phase I is applied. Hence, other than in Phase I, individual cuts have been applied to each detector accounting for the increased noise.

The low A/E cut is chosen according to $90 \%$ acceptance of the DEP events. Figure 23 shows for each BEGe detector and its respective $\mathrm{A} / \mathrm{E}$ cuts the survival fractions for various energy and peak regions in the ${ }^{228} \mathrm{Th}$ spectrum. The survival fractions in the peak and Compton regions increase from top to bottom in the respective detector strings, reflecting the deterioration of the $\mathrm{A} / \mathrm{E}$ resolution from top to bottom. For further suppression of surface events, a high $\mathrm{A} / \mathrm{E}$ cut with twice the separation from $\mathrm{A} / \mathrm{E}=1$ as the lower cut is applied.

Table 11 provides a summary from the combined data of all BEGe detectors and calibration runs. The survival fraction of Compton events from ${ }^{208} \mathrm{Tl}$ is $45.3 \%$ at $Q_{\beta \beta}$. Full energy $\gamma$ peaks (FEPs) are suppressed to less than $15 \%$. The strongest suppression (11\%) is achieved for single escape peaks (SEPs) which exhibit a relatively large MSE component. The high $\mathrm{A} / \mathrm{E}$ cut reduces the survival fractions of events in the DEP and $Q_{\beta \beta}$ region by less than $5 \%$.

\subsection{LAr veto}

In ultra-pure LAr the scintillation light yield is about $41 \times 10^{3}$ photons per $1 \mathrm{MeV}$ electron-equivalent energy deposition for
Table 11 Rejected event fractions by the low and high A/E cuts, and the survival fractions (s.f.) from all BEGe detectors and calibration runs [37]

\begin{tabular}{llll}
\hline Region & $\begin{array}{l}\text { Low cut } \\
(\%)\end{array}$ & $\begin{array}{l}\text { High cut } \\
(\%)\end{array}$ & $\begin{array}{l}\text { s.f. } \\
(\%)\end{array}$ \\
\hline DEP $(1593)$ & $10.0(2)$ & $2.68(6)$ & $87.3(2)$ \\
FEP $(1621)$ & $83.5(3)$ & $1.62(8)$ & $14.9(3)$ \\
FEP $(2615)$ & $83.58(3)$ & $1.82(1)$ & $14.60(2)$ \\
SEP $(2104)$ & $87.8(2)$ & $1.55(4)$ & $10.6(2)$ \\
$(2039 \pm 35) \mathrm{keV}$ & $52.5(1)$ & $2.20(2)$ & $45.3(1)$ \\
\hline
\end{tabular}

the fast and slow (triplet) component of the Ar excimer [38]. The purity of LAr has a strong influence on the light yield affecting predominantly the light emitted from the triplet state whose lifetime can thus be taken as a good indication for the LAr purity. For ultra-pure LAr, it is 1590(100) ns [39]. The triplet lifetime of the LAr contained in the GERDA cryostat has been deduced by averaging the waveforms (see Fig. 18) acquired with the PMTs of the LAr veto system and fitting the slow exponential tail (Fig. 24). The fit yields a value of 968(11)ns, indicating a still acceptable LAr purity after almost 6 years of operation without a significant refill and any purification.

The LAr veto system is running stably with all PMT and SiPM channels fully functional since March 2016. Before this date, 2 PMTs were not operational due to contact problems outside the lock system. During commissioning the LAr veto system has been immersed into the cryostat (and warmed up) more than 20 times. No ageing effects have been observed since its first deployment in April 2015.

The dark rate in the individual PMTs and SiPM channels is $300-500 \mathrm{~Hz}$ and $<200 \mathrm{~Hz}$, respectively. It is mostly due to the $\beta$ decay $\left(\mathrm{Q}_{\beta}=565 \mathrm{keV}\right)$ of ${ }^{39} \mathrm{Ar}\left(8 \times 10^{-16}\right.$ abundance relative to the stable ${ }^{40} \mathrm{Ar}$ ) with an activity of $\sim 1 \mathrm{~Bq} / \mathrm{kg}$ of ${ }^{40} \mathrm{Ar}$ [40]. The resulting dead time is about $2 \%$. Note that the radon shroud [5] limits the field of view of the SiPM-fiber 


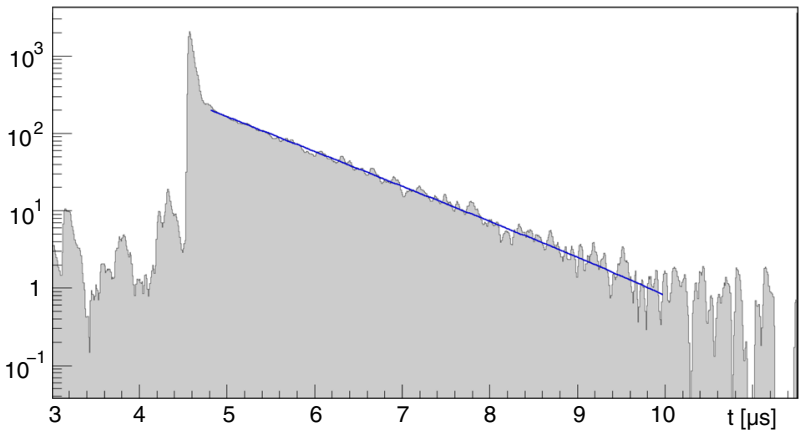

Fig. 24 Average PMT waveform recorded on 30.09.2015 in the LAr of the GERDA cryostat. The solid line indicates the triplet lifetime fit with a decay time of $0.97 \mu$ s [26]

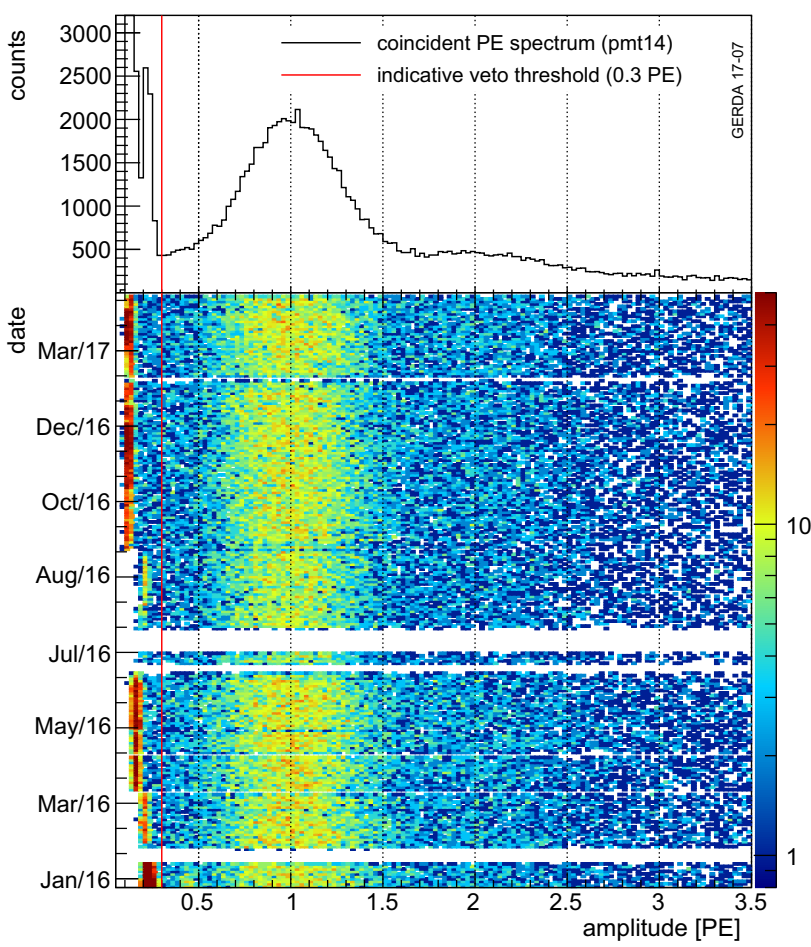

Fig. 25 Pulse height spectrum of a PMT (top) deduced from the scatter plot (bottom) which has been accumulated between January 2016 and April 2017

assembly in the cryostat to radii of less than $37.5 \mathrm{~cm}$; our preliminary estimate for the attenution length is $\sim 15 \mathrm{~cm}$.

The stability and noise level of the LAr veto system is continuously monitored with physics data. Figures 25 and 26 show on top a typical pulse height spectrum for a PMT and SiPM channel, respectively; and at bottom the scatter plots from which these pulse height spectra have been deduced. These data have been accumulated in coincidence with $\mathrm{Ge}$ triggers during a time span of about 16 months. The noise levels and gains are obviously stable, and the clear separation between noise and single photoelectron (PE) peak leads to a straightforward setting of the veto thresholds, indicated by

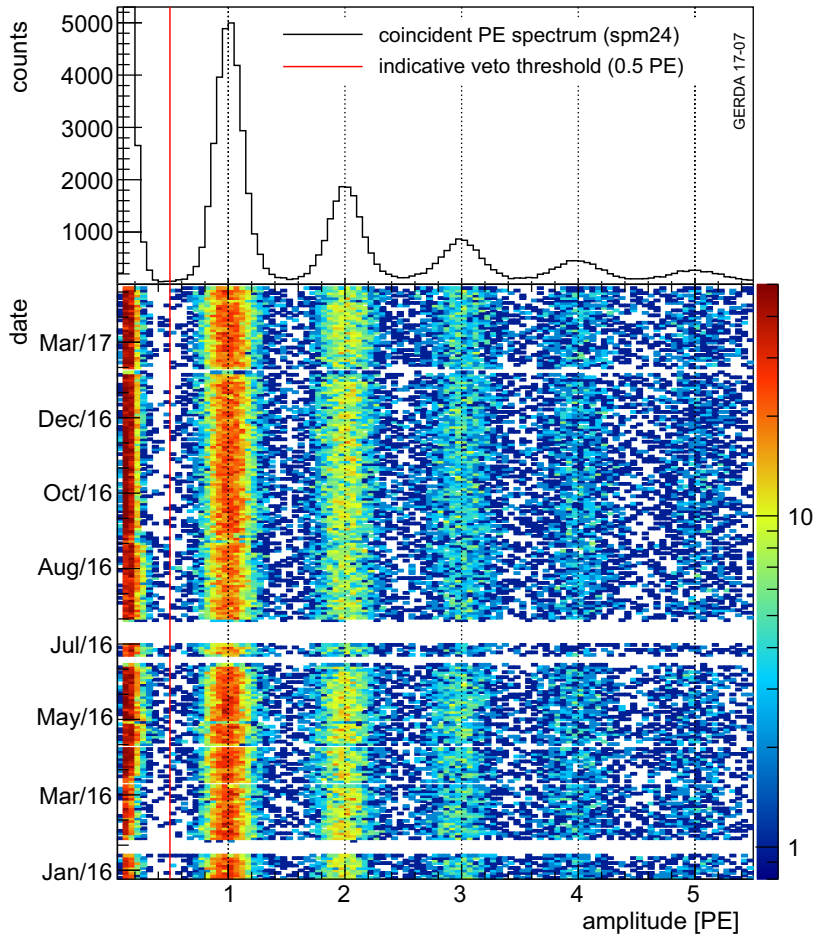

Fig. 26 Same as Fig. 25 but for a SiPM channel representing 6 SiPMs read out in parallel

the red lines, that can be easily readjusted if the gain jumps. These settings are 0.2-0.35 PE for PMTs, and 0.4-0.6 PE for SiPMs; the threshold is higher for only one PMT channel (0.6 PE) and one SiPM channel (0.9PE) where the individual SiPMs exhibit slightly different gains.

The performance of the LAr veto system has been tested with both a ${ }^{228} \mathrm{Th}$ and ${ }^{226} \mathrm{Ra}$ source. Figure 27 shows the measured spectra as obtained after standard quality cuts, and after indicated additional cuts. Both spectra exhibit the same specific features that have been observed in the LARGE test stand; for a detailed discussion see [22]. One highlight is displayed in the inset of the ${ }^{228} \mathrm{Th}$ spectrum (Fig. 27, 1.h.s.). The DEP of the ${ }^{208} \mathrm{Tl} 2615 \mathrm{keV} \gamma$ line at $1593 \mathrm{keV}$ is barely suppressed by PSD $(\approx 90 \%$ acceptance) while the LAr veto cut eliminates it completely due to the two escaping $511 \mathrm{keV}$ annihilation quanta. On the other hand, the ${ }^{212} \mathrm{Bi}$ single $\gamma$ line at $1621 \mathrm{keV}$ is largely accepted by the LAr veto because the full energy is deposited in the Ge detector.

The ${ }^{208} \mathrm{Tl} 2615 \mathrm{keV} \gamma$ line as well as the various ${ }^{214} \mathrm{Bi} \gamma$ lines in the ${ }^{226} \mathrm{Ra}$ spectrum (Fig. 27, r.h.s.) provide significant contributions to the background around $Q_{\beta \beta}$ [12], and hence the suppression of the continuum around $Q_{\beta \beta}$ is of special interest. The respective suppression factors are collected in Table 12. While the suppression by PSD is comparable, a much stronger suppression by the LAr veto for ${ }^{228} \mathrm{Th}$ than for ${ }^{226} \mathrm{Ra}$ is noticed. This can be understood since for ${ }^{226} \mathrm{Ra}$ the main contribution to the Compton continuum is due to 


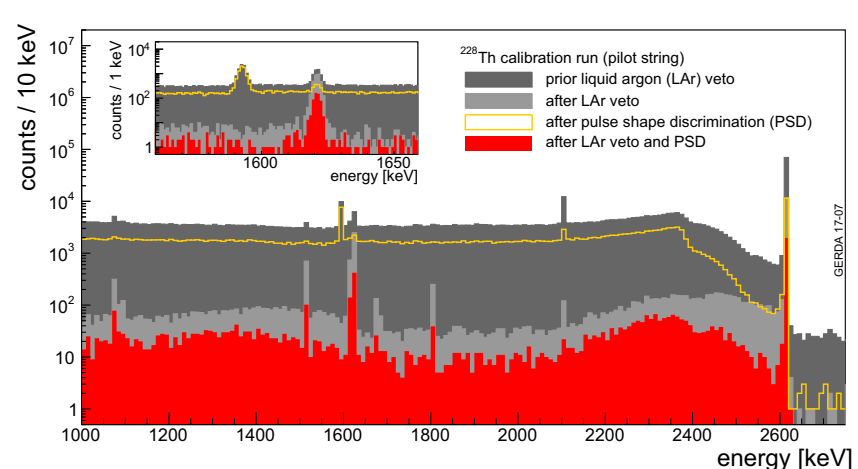

Fig. 27 Calibration spectra for ${ }^{228} \mathrm{Th}$ (left) and ${ }^{226} \mathrm{Ra}$ (right) with detector anti-coincidence and muon veto (dark grey), with suppression by the LAr veto system and pulse shape discrimination (PSD), and by the combination of LAr veto system and PSD. The inset on the 1.h.s.

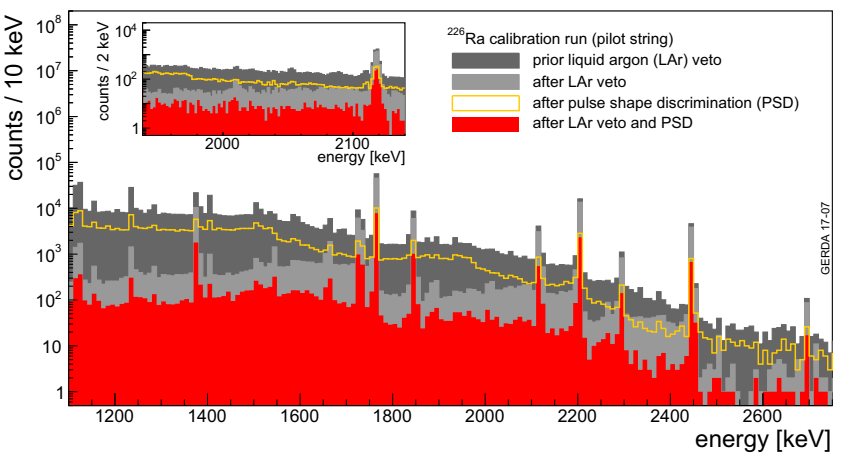

shows the double escape peak of ${ }^{208} \mathrm{Tl}$ at $1593 \mathrm{keV}$ and the $1621 \mathrm{keV}$ single gamma line of ${ }^{212} \mathrm{Bi}$, on the r.h.s the $2118 \mathrm{keV}$ single gamma line of ${ }^{214} \mathrm{Bi}$

Table 12 Suppression factors obtained in the ROI with a ${ }^{228} \mathrm{Th}$ and ${ }^{226} \mathrm{Ra}$ source from Ge anti-coincidences (anti), the LAr veto (LAr), pulse shape discrimination (PSD), and after all cuts (all); the acceptance (acc.) values refer to all cuts

\begin{tabular}{llllll}
\hline Source & Anti & LAr & PSD & All & Acc. \\
\hline${ }^{228} \mathrm{Th}$ & $1.26(1)$ & $98(4)$ & $2.19(1)$ & $345(25)$ & 0.868 \\
${ }^{226} \mathrm{Ra}$ & $1.26(1)$ & $5.7(2)$ & $2.98(6)$ & $29(3)$ & 0.899 \\
\hline
\end{tabular}

the $2204 \mathrm{keV}$ single $\gamma$ line so that less than $200 \mathrm{keV}$ are available for deposition in the $\mathrm{LAr}$; in case of ${ }^{228} \mathrm{Th}$, however, the more energetic $2615 \mathrm{keV} \gamma$ ray is accompanied in $86 \%$ of the cases by a $583 \mathrm{keV} \gamma$ ray so that more energy is available for deposition in the LAr. In general, our measured suppression factors are smaller than the ones obtained in LARGE [22]. This might be due to several reasons, e.g. different geometry with different shadowing conditions, different radioactive sources with different leakage of $\beta$ particles, and, definitely, lower purity of the LAr.

The performance of the LAr veto system can be monitored continuously with the two strongest $\gamma$ lines of the physics data (Fig. 28, see also Fig. 30). The $\gamma$ line at $1525 \mathrm{keV}$ is due to the decay of ${ }^{42} \mathrm{~K}$, a $\beta-\gamma$ cascade, in which the $\beta$ particle can deposit up to $2 \mathrm{MeV}$ in the LAr. The LAr veto system suppresses this line by typically a factor of 5 . On the other hand, the ${ }^{40} \mathrm{~K}$ line at $1461 \mathrm{keV}$, a single $\gamma$ line, is not suppressed since it follows electron capture of ${ }^{40} \mathrm{~K}$ without any energy deposition in the LAr. Hence, no suppression is expected apart from random coincidences so that this line can be used to determine the LAr veto acceptance independently of the pulser.

\subsection{Muon veto system}

Figure 29 shows for 16 months in 2016/17 the daily rate of one PMT of each of the seven PMT rings in the water tank [5], i.e. of varying height in the water. Only the standard

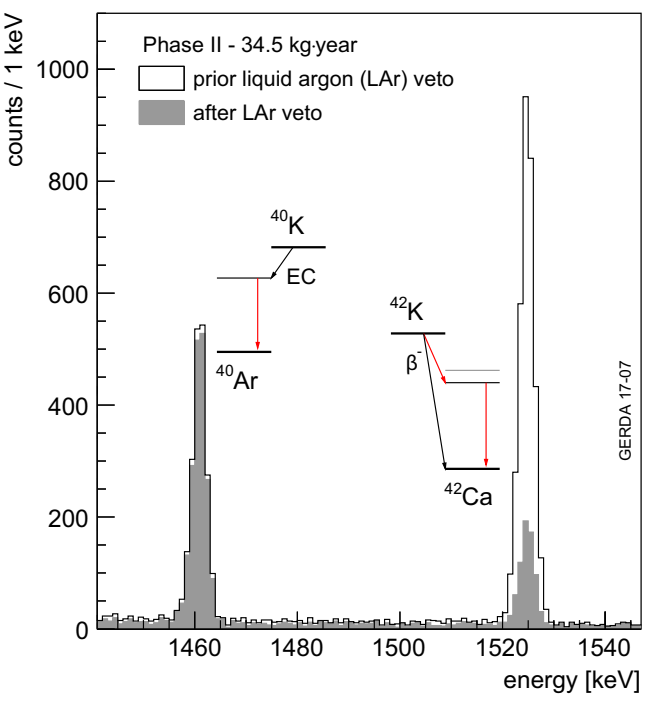

Fig. 28 Monitoring the LAr veto performance with the ${ }^{40} \mathrm{~K}$ and ${ }^{42} \mathrm{~K} \gamma$ lines (see text)

trigger requirements are requested: either 5 Cherenkov PMTs within $60 \mathrm{~ns}$ with a signal above threshold of $0.5 \mathrm{PE}$, or a triple coincidence within the plastic veto. A mean stability of $4 \%$ can be observed. No readjustment of the HV was necessary during the 477 days of operation of Phase II. The mean daily rate of 3164(6) muons was measured which translates into a rate of $3.54 \times 10^{-4} /\left(\mathrm{m}^{2} \mathrm{~s}\right)$. This is a $1.8 \%$ deviation from the mean of Phase I [42], still consistent and giving hints on systematic uncertainties. 


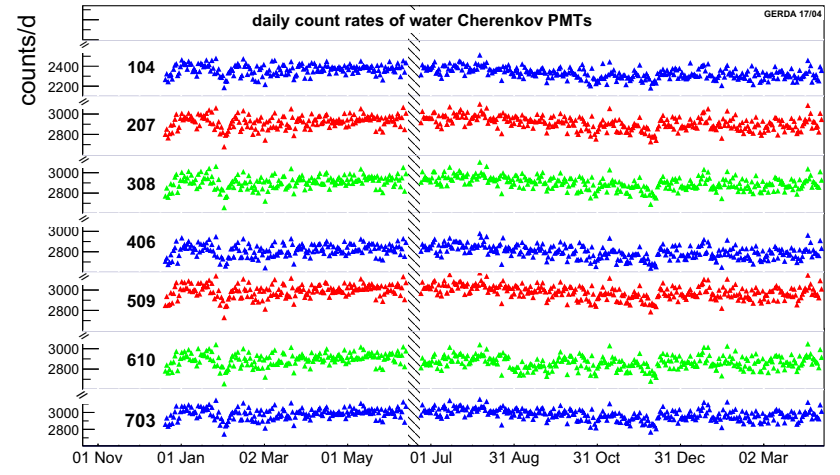

Fig. 29 Daily rates of water Cherenkov PMTs, one from each ring (c.f. Ref. [41])

A further proof of the reliability of the muon veto performance comes through the analysis of the muon seasonal variation. Despite the shorter measurement period essentially the same parameters were derived as previously [42].

\subsection{Background levels}

\subsubsection{Spectra}

Figure 30 shows the exposure-normalized Phase II background spectra obtained with the BEGe and enriched semicoaxial detectors after quality cuts but before LAr veto and PSD. Both spectra agree well, exhibiting the same prominent features as observed in Phase I: below $500 \mathrm{keV}$ the tail of the ${ }^{39} \mathrm{Ar} \beta$-spectrum, between 600 and $1600 \mathrm{keV}$ the broad structure from the $2 \nu \beta \beta$ decays, individual $\gamma$-lines between 400 and $2650 \mathrm{keV}$, and $\alpha$-structures above $3500 \mathrm{keV}$ predominantly due to ${ }^{210} \mathrm{Po}$ and ${ }^{226} \mathrm{Ra}$ decays.
The observed differences for the tail of the ${ }^{39} \mathrm{Ar} \beta$ spectrum originate from the different dead layers in BEGe and coaxial detectors, in particular from the large and thin $\mathrm{p}^{+}$contact in the bore hole of the latter detector type.

The pulse shapes of the observed $\alpha$-events reveal them to be predominantly located at the surface of the $\mathrm{p}^{+}$contact. While the count rate is relatively low and similar in case of the BEGe detectors, it is larger for the coaxial ones where one detector (ANG4) contributes more than 50\% to the total rate. The origin of the contamination is not understood, but there is evidence that it occurred during the fabrication process.

\subsubsection{Intensity of $\gamma$ lines}

The intensities of the $\gamma$ lines carry information about the nature and the location of various contamination sources [12]. While the detailed model study of the background is in progress, we note here that the count rates of the ${ }^{40} \mathrm{~K}$ and ${ }^{42} \mathrm{~K}$ lines are increased strongly in Phase II, by factors of 4 and 2 compared to Phase I, respectively. Since the lines are located well below $\mathrm{Q}_{\beta \beta}$ they will not affect the background index. The increased ${ }^{40} \mathrm{~K}$ intensity might be due to the different kind and increased number of cables which run in Phase II along the detector array as well as to the LAr veto system. The increased ${ }^{42} \mathrm{~K}$ intensity is understood qualitatively by the change from metallic to plastic mini-shrouds. The latter no longer shield the electric fields of the Ge detectors so that more ${ }^{42} \mathrm{~K}$ ions can be attracted closer to the detectors. For all other $\gamma$ lines the count rates are similar to those observed in Phase I; the intensities of the ${ }^{214} \mathrm{Bi} \gamma$-lines is even lower than in Phase I. This is a remarkable success considering the additional amount of material that has been deployed in Phase II close to the detector array.

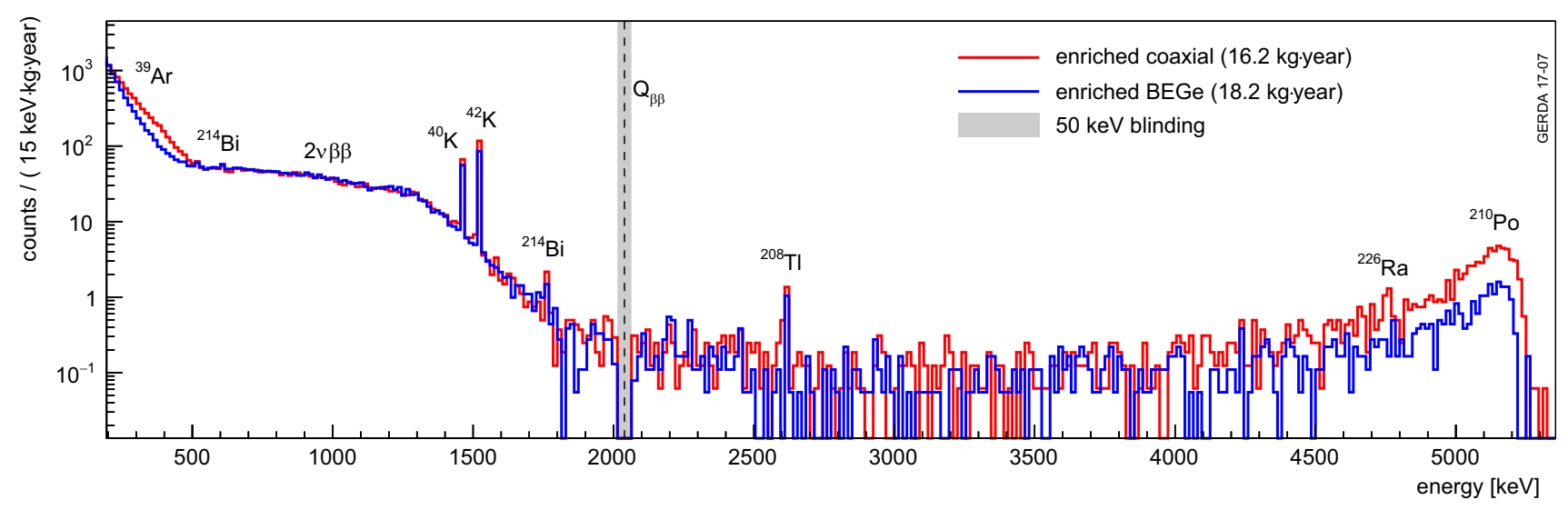

Fig. 30 Normalized GERDA Phase II background spectra from BEGe and enriched coaxial detectors before pulse shape discrimination and LAr veto. The grey bar indicates the blinded energy region around $Q_{\beta \beta}$ 
Table 13 Counts in the analysis window observed in Phase IIa and IIb with indicated exposures $\mathcal{E}$ after anti-coincidences within the detector array (AC), after anti-coincidences and LAr veto (LAr) or pulse shape discrimination (PSD), and after all cuts (all). The quoted background

\begin{tabular}{lllllll}
\hline Det/phase & $\begin{array}{l}\mathcal{E} \\
\text { (kgyear) }\end{array}$ & $\begin{array}{l}\text { AC } \\
\text { cts }\end{array}$ & $\begin{array}{l}\text { LAr } \\
\text { cts }\end{array}$ & $\begin{array}{l}\text { PSD } \\
\text { cts }\end{array}$ & $\begin{array}{l}\text { all } \\
\text { cts }\end{array}$ & $\begin{array}{l}\text { BI } \\
\text { (biu) }\end{array}$ \\
\hline $\begin{array}{c}\text { coax/IIa } \\
\text { /IIb* }\end{array}$ & 5.0 & 19 & 12 & 8 & 4 & $3.5_{-1.5}^{+2.1}$ \\
Total* & 11.2 & 38 & 14 & 17 & 5 & 9 \\
\hline BEGe/IIa & 16.2 & 57 & 26 & 25 & 1 \\
/IIb & 5.8 & 21 & 6 & 6 & 3 & $0.7_{-0.8}^{+1.0}$ \\
total & 12.4 & 30 & 11 & 11 & 4 & $1.0_{-0.4}^{+0.6}$ \\
\hline
\end{tabular}

*Values preliminary since PSD cuts are not yet finalized

\subsubsection{Background index at $Q_{\beta \beta}$}

Table 13 collects data in the analysis window ${ }^{8}$ around $Q_{\beta \beta}$ which are used to extract the BI in Phase II. In addition, to the Phase IIa data (December 2015-June 2016) of the first data release [3], data of subsequent physics runs until April 2017 (Phase IIb) are shown which exhibit a more than 50\% larger exposure. These data with higher statistics confirm the BI reported from the first data release with increased significance yielding in total $\left(2.7_{-0.8}^{+1.1}\right) \times 10^{-3} \mathrm{cts} /(\mathrm{keV} \mathrm{kg}$ year $)$ for the coaxial and $\left(1.0_{-0.4}^{+0.6}\right) \times 10^{-3} \mathrm{cts} /(\mathrm{keV} \mathrm{kg}$ year) for the BEGe detectors. This corroborates that the goal of the Phase II upgrade, an improvement of the BI by a factor of 10 compared to Phase I, has been achieved.

Table 13 shows also the counts in the analysis window after various cuts. After anti-coincidences within the detector array these counts correspond to a BI below (1-2) $\times 10^{-2} \mathrm{cts} /(\mathrm{keV} \mathrm{kg}$ year) for both BEGe and coaxial detectors. Within the limited statistics, the LAr veto and PSD seem to suppress background events with comparable strength. As first evidence for various contributing suppression mechanisms it is noted that of the 108 events surviving the anticoincidence cut, 23 resp. 30 events are cut exclusively by the LAr veto and PSD, while 42 events are cut by both LAr veto and PSD.

\section{Conclusion}

GERDA continues to exploit novel technologies in order to study neutrinoless double beta decay of ${ }^{76} \mathrm{Ge}$ with unprecedented low background rate. Operating in Phase I for the

\footnotetext{
8 The analysis window extends from 1930 to $2190 \mathrm{keV}$ excluding the intervals 2104(5) and 2119(5) keV of known $\gamma$ lines from ${ }^{208} \mathrm{Tl}$ (SEP) and ${ }^{214} \mathrm{Bi}$. In addition, the $50 \mathrm{keV}$ wide blinded interval centered at $Q_{\beta \beta}$ is excluded for the Phase IIb coaxial data which are not yet unblinded.
}

indices $\mathrm{BI}$ in units (biu) of $10^{-3} \mathrm{cts} /(\mathrm{keV} \mathrm{kg}$ year) have been determined after all cuts; the uncertainties are statistical and deduced for a Poissonian signal 
combined data from these calibrations runs are used to optimize event selection criteria and efficiencies of pulse shape discrimination. The performance of the LAr veto system has been established by dedicated calibrations runs with ${ }^{228} \mathrm{Th}$ and ${ }^{226} \mathrm{Ra}$ sources yielding suppression factors at $Q_{\beta \beta}$ of about 100 and 6, respectively. Quasi-continuous monitoring during the physics runs is achieved by analysis of the relatively strong ${ }^{40} \mathrm{~K}$ and ${ }^{42} \mathrm{~K} \gamma$ lines at $1461 \mathrm{keV}$ and $1525 \mathrm{keV}$.

Based on an exposure of $10.8 \mathrm{~kg}$ year results of a first period of the Phase II physics run have been published recently [3]. They demonstrate that the goal of Phase II, a background index of about $10^{-3} \mathrm{cts} /(\mathrm{keV} \mathrm{kg}$ year), has been achieved. This finding is statistically corroborated here with data from a more than doubled exposure of $23.6 \mathrm{~kg}$ year. GERDA will thus remain "background-free" up to its design exposure of $100 \mathrm{~kg}$ year reaching thereby a sensitivity beyond $10^{26}$ year.
Acknowledgements The GERDA experiment is supported by the German Federal Ministry for Education and Research (BMBF), the German Research Foundation (DFG) via the Excellence Cluster Universe, the Italian Istituto Nazionale di Fisica Nucleare (INFN), the Max Planck Society (MPG), the Polish National Science Centre (NCN), the Foundation for Polish Science (TEAM/2016-2/17), the Russian Foundation for Basic Research (RFBR) and the Swiss National Science Foundation (SNF). These research institutions acknowledge internal financial support.

This project has received funding/support from the European Union's Horizon 2020 research and innovation programme under the Marie Sklodowska-Curie grant agreements No 690575 and No 674896.

The GERDA Collaboration thanks the directors and the staff of the LNGS for their support of the GERDA experiment.

Open Access This article is distributed under the terms of the Creative Commons Attribution 4.0 International License (http://creativecomm ons.org/licenses/by/4.0/), which permits unrestricted use, distribution, and reproduction in any medium, provided you give appropriate credit to the original author(s) and the source, provide a link to the Creative Commons license, and indicate if changes were made. Funded by SCOAP ${ }^{3}$.

\section{Appendix}

See Table 14 and Figs. 31, 32, 33, 34.

increases from top to bottom. The active masses of the newly produced BEGe detectors include a correction that considers a full charge collection depth growth occurred during storage at room temperature in the three years before deployment in GERDA. Finally, the full energy peak detector efficiencies $\epsilon_{f e p}$ for the $0 \nu \beta \beta$ decay in ${ }^{76} \mathrm{Ge}$ are quoted. For

discussion of errors see Sec. 2.1.2
Table 14 Main parameters of all detectors used in GERDA Phase II. The GD, ANG and RG detectors are made of germanium enriched in ${ }^{76} \mathrm{Ge}$ from 85.5 to $88.3 \%$. The three GTF detectors are made from natural germanium. The operational voltages recommended by the manufacturer are quoted. A ' $y$ ' marks in column 4 the detectors with a passivation layer (PL) in the groove. The position number in a given string

\begin{tabular}{|c|c|c|c|c|c|c|c|c|}
\hline Nr. & detector & $\mathrm{V}_{\text {rec }}[\mathrm{kV}]$ & with PL & string \& position & $f_{G e 76}$ & $M_{\text {diode }}[\mathrm{g}]$ & $\left.M_{a v}{ }_{-u c o r r-c o r r}^{+u c o r r}+\mathrm{g}\right]$ & $\epsilon_{f e p} \pm$ ucorr \pm corr $r$ \\
\hline 13 & GD32A & 3.0 & & III-2 & $0.877 \pm 0.013$ & 458 & $404_{-10-2}^{+10+4}$ & $0.888 \pm 0.001 \pm 0.002$ \\
\hline 12 & GD32B & 4.0 & & III-1 & $0.877 \pm 0.013$ & 716 & $632_{-10-2}^{+10+4}$ & $0.900 \pm 0.001 \pm 0.002$ \\
\hline 14 & GD32C & 4.0 & & III-3 & $0.877 \pm 0.013$ & 743 & $665_{-10-2}^{+10+4}$ & $0.901 \pm 0.001 \pm 0.002$ \\
\hline 34 & GD32D & 4.0 & & VI-4 & $0.877 \pm 0.013$ & 720 & $657_{-10-2}^{+10+5}$ & $0.900 \pm 0.001 \pm 0.002$ \\
\hline 24 & GD35A & 4.0 & & IV-5 & $0.877 \pm 0.013$ & 768 & $693_{-13-2}^{+13+3}$ & $0.904 \pm 0.001 \pm 0.002$ \\
\hline 1 & GD35B & 4.0 & & $\mathrm{I}-1$ & $0.877 \pm 0.013$ & 810 & $740_{-11-2}^{+11+5}$ & $0.902 \pm 0.001 \pm 0.002$ \\
\hline 19 & GD35C & 3.5 & $\mathrm{y}$ & IV-0 & $0.877 \pm 0.013$ & 634 & $572_{-9-3}^{+9+4}$ & $0.893 \pm 0.001 \pm 0.002$ \\
\hline 4 & GD61A & 4.5 & $\mathrm{y}$ & $\mathrm{I}-4$ & $0.877 \pm 0.013$ & 731 & $652_{-11-3}^{+12+4}$ & $0.902 \pm 0.001 \pm 0.002$ \\
\hline 26 & GD61B & 4.0 & $\mathrm{y}$ & IV-7 & $0.877 \pm 0.013$ & 751 & $666_{-12-2}^{+12+5}$ & $0.899 \pm 0.001 \pm 0.002$ \\
\hline 16 & GD61C & 4.0 & & III-5 & $0.877 \pm 0.013$ & 634 & $562_{-10-3}^{+10+5}$ & $0.892 \pm 0.001 \pm 0.002$ \\
\hline 17 & GD76B & 3.5 & $\mathrm{y}$ & III-6 & $0.877 \pm 0.013$ & 384 & $326_{-7-2}^{+7+3}$ & $0.883 \pm 0.001 \pm 0.002$ \\
\hline 20 & GD76C & 3.5 & $\mathrm{y}$ & IV-1 & $0.877 \pm 0.013$ & 824 & $723_{-12-2}^{+12+5}$ & $0.902 \pm 0.001 \pm 0.002$ \\
\hline 32 & GD79B & 3.5 & & VI-2 & $0.877 \pm 0.013$ & 736 & $648_{-13-2}^{+13+5}$ & $0.897 \pm 0.001 \pm 0.002$ \\
\hline 23 & GD79C & 3.5 & & IV-4 & $0.877 \pm 0.013$ & 812 & $713_{-11-2}^{+11+5}$ & $0.900 \pm 0.001 \pm 0.002$ \\
\hline 35 & GD89A & 4.0 & & VI-5 & $0.877 \pm 0.013$ & 524 & $462_{-9-2}^{+10+3}$ & $0.893 \pm 0.001 \pm 0.002$ \\
\hline 5 & GD89B & 3.5 & $\mathrm{y}$ & $\mathrm{I}-5$ & $0.877 \pm 0.013$ & 620 & $533_{-12-2}^{+12+4}$ & $0.890 \pm 0.001 \pm 0.002$ \\
\hline 15 & GD89C & 4.0 & $\mathrm{y}$ & III-4 & $0.877 \pm 0.013$ & 595 & $520_{-11-2}^{+12+5}$ & $0.889 \pm 0.001 \pm 0.002$ \\
\hline 21 & GD89D & 4.0 & & IV-2 & $0.877 \pm 0.013$ & 526 & $454_{-9-2}^{+9+5}$ & $0.884 \pm 0.001 \pm 0.002$ \\
\hline
\end{tabular}


Table 14 continued

\begin{tabular}{|c|c|c|c|c|c|c|c|c|}
\hline Nr. & detector & $\mathrm{V}_{\text {rec }}[\mathrm{kV}]$ & with PL & string \& position & $f_{G e 76}$ & $M_{\text {diode }}[\mathrm{g}]$ & $M_{a v}{ }_{-u c o r r}^{+ \text {ucorr }}[\mathrm{g}]$ & $\epsilon_{\text {fep }} \pm$ ucorr \pm corr \\
\hline 0 & GD91A & 3.5 & & $\mathrm{I}-0$ & $0.877 \pm 0.013$ & 627 & $557_{-11-2}^{+10+3}$ & $0.898 \pm 0.001 \pm 0.002$ \\
\hline 25 & GD91B & 3.5 & & IV-6 & $0.877 \pm 0.013$ & 650 & $578_{-10-2}^{+10+5}$ & $0.897 \pm 0.001 \pm 0.002$ \\
\hline 7 & GD91C & 4.0 & $\mathrm{y}$ & $\mathrm{I}-7$ & $0.877 \pm 0.013$ & 627 & $556_{-11-2}^{+11+4}$ & $0.896 \pm 0.001 \pm 0.002$ \\
\hline 33 & GD91D & 4.5 & & VI-3 & $0.877 \pm 0.013$ & 693 & $615_{-12-2}^{+12+5}$ & $0.899 \pm 0.001 \pm 0.002$ \\
\hline 30 & GD00A & 2.5 & $\mathrm{y}$ & VI-0 & $0.877 \pm 0.013$ & 496 & $439_{-9-2}^{+8+3}$ & $0.888 \pm 0.001 \pm 0.002$ \\
\hline 3 & GD00B & 3.5 & & $\mathrm{I}-3$ & $0.877 \pm 0.013$ & 697 & $613_{-12-2}^{+12+5}$ & $0.897 \pm 0.001 \pm 0.002$ \\
\hline 18 & GD00C & 3.5 & $\mathrm{y}$ & III-7 & $0.877 \pm 0.013$ & 815 & $727_{-13-2}^{+14+5}$ & $0.903 \pm 0.001 \pm 0.002$ \\
\hline 22 & GD00D & 3.5 & $\mathrm{y}$ & IV-3 & $0.877 \pm 0.013$ & 813 & $723_{-13-2}^{+13+5}$ & $0.902 \pm 0.001 \pm 0.002$ \\
\hline 11 & GD02A & 2.5 & $\mathrm{y}$ & III-0 & $0.877 \pm 0.013$ & 545 & $488_{-8-2}^{+8+3}$ & $0.893 \pm 0.001 \pm 0.002$ \\
\hline 2 & GD02B & 3.0 & & $\mathrm{I}-2$ & $0.877 \pm 0.013$ & 625 & $553_{-10-2}^{+10+4}$ & $0.895 \pm 0.001 \pm 0.002$ \\
\hline 31 & GD02C & 3.5 & & VI-1 & $0.877 \pm 0.013$ & 788 & $700_{-13-2}^{+13+5}$ & $0.901 \pm 0.001 \pm 0.002$ \\
\hline 6 & $\mathrm{GD}_{02 \mathrm{D}}^{a}$ & 4.0 & $\mathrm{y}$ & $\mathrm{I}-6$ & $0.877 \pm 0.013$ & 662 & $552_{-11-2}^{+11+0}$ & Not defined, see remark \\
\hline 36 & ANG1 & 4.0 & & VI-6 & $0.859 \pm 0.029$ & 958 & $795_{-43-26}^{+43+26}$ & $0.889 \pm 0.018$ \\
\hline 27 & ANG2 & 4.0 & $\mathrm{y}$ & $\mathrm{V}-0$ & $0.866 \pm 0.025$ & 2833 & $24688_{-121-80}^{+121+80}$ & $0.918 \pm 0.018$ \\
\hline 10 & ANG3 & 3.5 & $\mathrm{y}$ & II-2 & $0.883 \pm 0.026$ & 2391 & $2070_{-118-67}^{+118+60}$ & $0.916 \pm 0.018$ \\
\hline 29 & ANG4 & 3.0 & $\mathrm{y}$ & $\mathrm{V}-2$ & $0.863 \pm 0.013$ & 2372 & $2136_{-116-69}^{+116+69}$ & $0.916 \pm 0.018$ \\
\hline 8 & ANG5 & 2.5 & & II-0 & $0.856 \pm 0.013$ & 2746 & $2281_{-109-74}^{+109+74}$ & $0.918 \pm 0.018$ \\
\hline 9 & RG1 & 5.0 & & II-1 & $0.855 \pm 0.015$ & 2110 & $1908_{-109-62}^{+109+62}$ & $0.915 \pm 0.018$ \\
\hline 28 & RG2 & 4.0 & & $\mathrm{~V}-1$ & $0.855 \pm 0.015$ & 2166 & $1800_{-99-58}^{+99+58}$ & $0.912 \pm 0.018$ \\
\hline 38 & GTF32 & 3.5 & $\mathrm{y}$ & VII-1 & $0.078 \pm 0.001$ & 2321 & $2251_{-116}^{+116}$ & $0.92 \pm 0.018$ \\
\hline 39 & GTF45_2 & 3.5 & & VII-2 & $0.078 \pm 0.001$ & 2312 & 1965 & $0.92 \pm 0.018$ \\
\hline 37 & GTF112 & 3.5 & $\mathrm{y}$ & VII-0 & $0.078 \pm 0.001$ & 2965 & 2522 & $0.92 \pm 0.018$ \\
\hline
\end{tabular}

Remarks:

${ }^{a}$ Detector GD02D does not deplete due to an unsuitable impurity concentration. The material is rather a pn junction than of p-type 


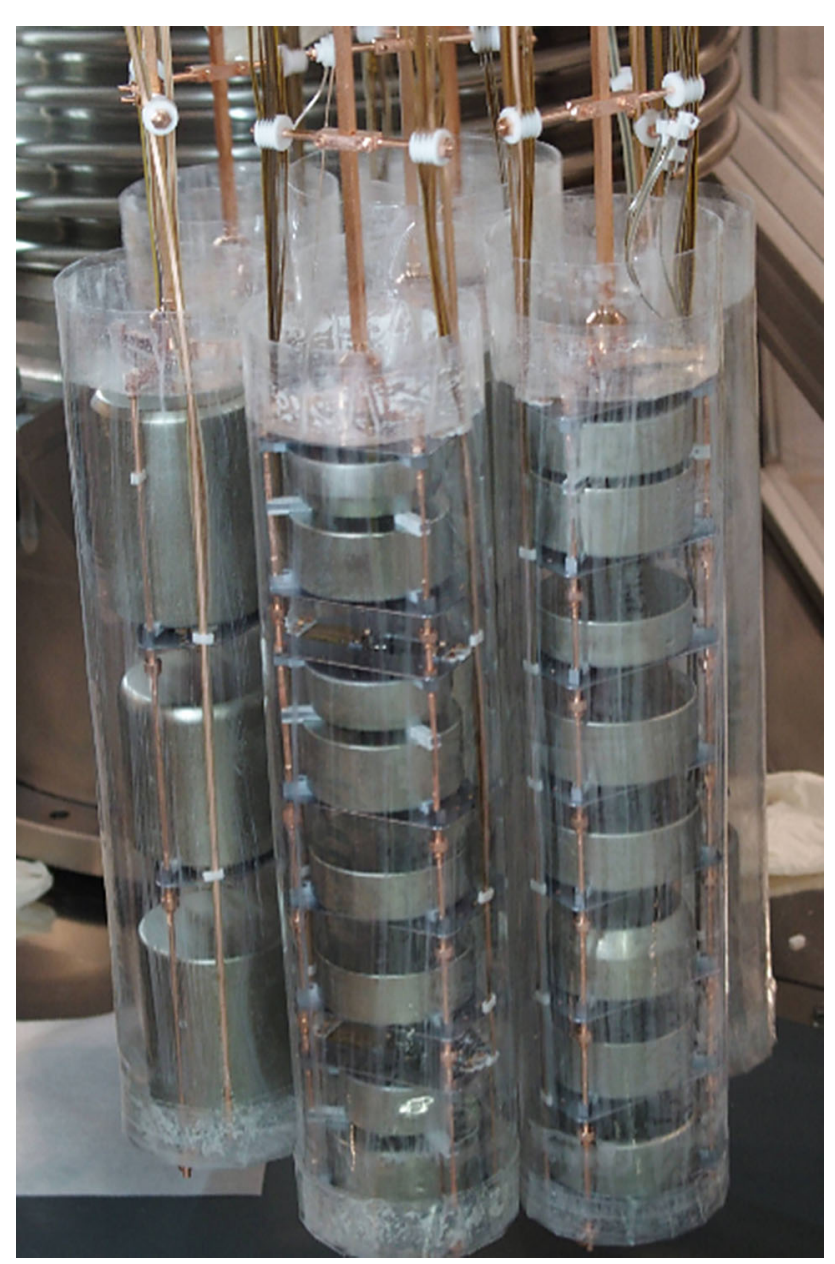

Fig. 31 Photo of GERDA Phase II detector array, showing from left to right string 2 with three semi-coaxial detectors, and strings 3 and 4 with eight BEGe detectors, respectively. Each of the seven strings is enclosed by a transparent mini-shroud

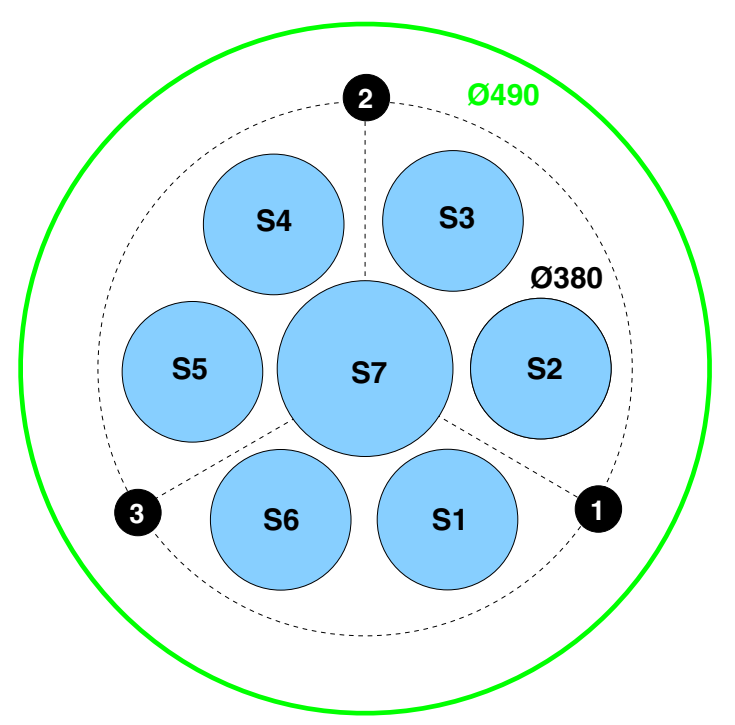

Fig. 32 View of the array from top. The positions of the three calibration sources $(1,2,3)$ and the boundaries of the LAr veto system $(\varnothing 490 \mathrm{~mm})$ are indicated. The surrounding radon shroud with a diameter of $750 \mathrm{~mm} \mathrm{[5]} \mathrm{is} \mathrm{not} \mathrm{shown}$ 
Fig. 33 Distribution of the enriched BEGe (GDxxx) and semi-coaxial (RGx and ANGx) detectors in the various strings of the GERDA Phase II detector array; the natural low-background semi-coaxial detectors carry the labels GTFxx. Blue colored detectors carry the manufacturer's passivation on the insulating groove between the $\mathrm{p}^{+}$and $\mathrm{n}^{+}$ contact; the yellow colored ones have this layer removed.

Horizontal grey lines indicate the positions of the silicon plates (see Fig. 3).

Detectors are numbered from top to bottom string by string, starting with nr. 0 in string 1

(GD91A) and ending with nr. 39 in string 7 (GTF45)

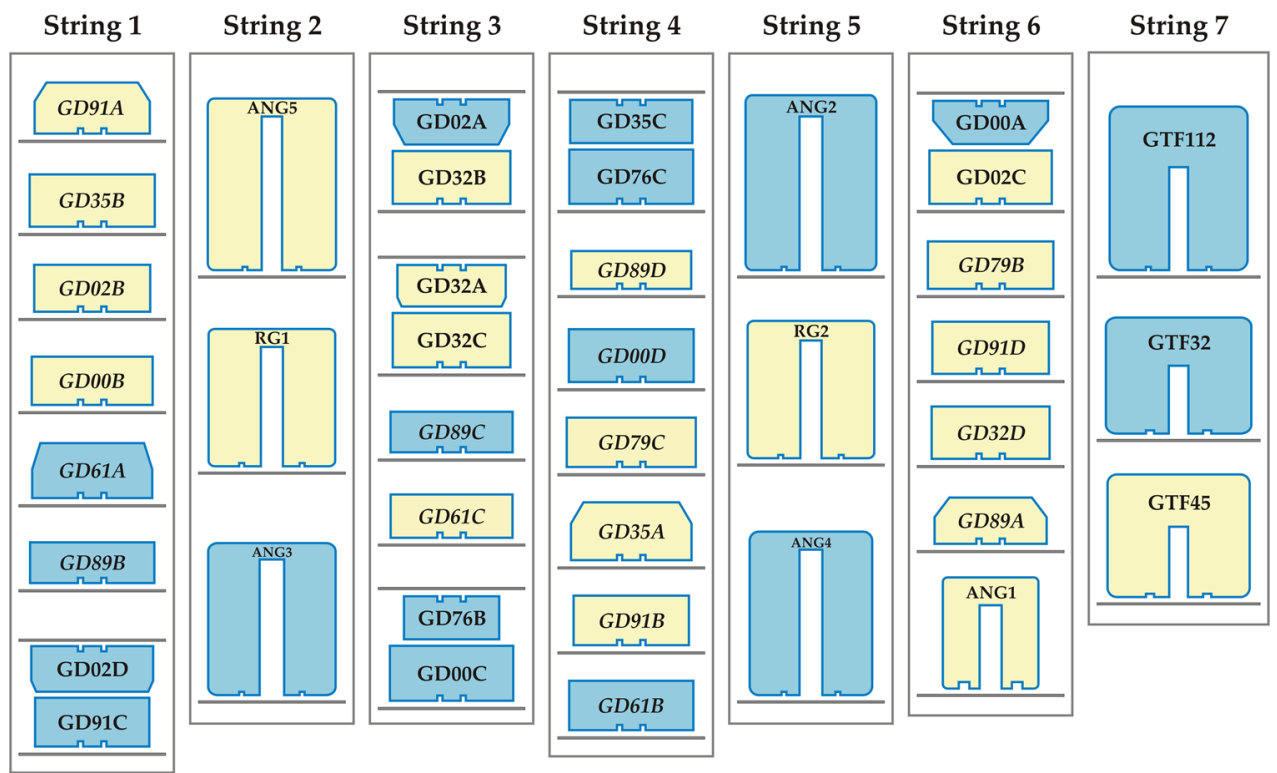


Fig. 34 Changes of leakage currents $\Delta(\mathrm{LC})$ of indicated detectors in the period from December 2015 to April 2017

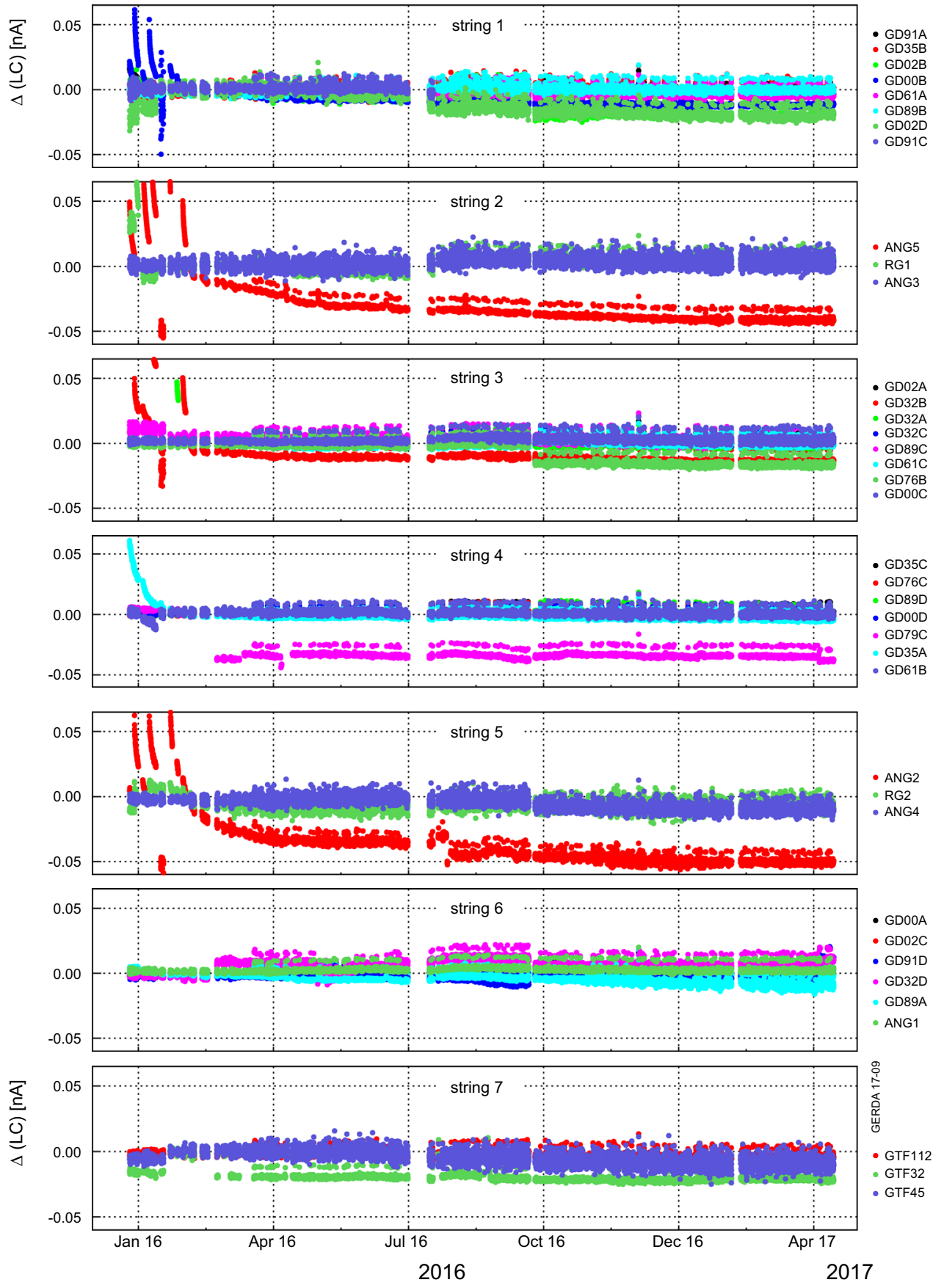




\section{References}

1. R.N. Mohapatra et al., Rep. Prog. Phys. 70, 1757 (2007)

2. C. Patrignani et al. (Particle Data Group), Chin. Phys. C 40, 100001 (2016) (p. 767 and update 2017)

3. M. Agostini et al. [Gerda collaboration], Nature 544, 47 (2017)

4. A. Gando et al. [KamLAND-Zen collaboration]. Phys. Rev. Lett. 117, 082503 (2016)

5. K.-H. Ackermann et al. [Gerda collaboration], Eur. Phys. J. C 73, 2330 (2013)

6. M. Giammarchi et al., AIP Conf. Proc. 1549, 209 (2013)

7. M. Agostini et al. [Gerda collaboration], Phys. Rev. Lett. 111, 122503 (2013)

8. H.V. Klapdor-Kleingrothaus, I.V. Krishoveina, A. Dietz, O. Chkvorets, Phys. Lett. B 586, 198 (2004)

9. H.V. Klapdor-Kleingrothaus, I.V. Krishoveina, Mod. Phys. Lett. A 21, 1547 (2006)

10. M. Agostini et al. [Gerda collaboration], Eur. Phys. J. C 75, 416 (2015)

11. M. Agostini et al. [Gerda collaboration], J. Phys. G Nucl. Part. Phys. 42, 115201 (2015)

12. M. Agostini et al. [Gerda collaboration], Eur. Phys. J. C 74, 2764 (2014)

13. M. Agostini et al. [Gerda collaboration], Eur. Phys. J. C 73, 2583 (2013)

14. M. Barnabe Heider, Dissertation, Universität Heidelberg (2009). https://doi.org/10.11588/heidok.00009551

15. M. Agostini et al. [Gerda collaboration], Eur. Phys. J. C 75, 39 (2015)

16. E. Andreotti et al., J. Instrum. 8, P06012 (2013)

17. T. Bode, Dissertation, Technische Universität München (2016)

18. S. Riboldi, A. Pullia, C. Cattadori, Nuclear Science Symposium and Medical Imaging Conference (NSS/MIC). IEEE, pp. 782 (2012). https://doi.org/10.1109/NSSMIC.2012.6551209

19. S. Riboldi et al., 4th International Conference on Advancements in Nuclear Instrumentation Measurement Methods and their Applications (ANIMMA, 2015), pp. 1. https://doi.org/10.1109/ANIMMA. 2015.7465549
20. A. Lubashevskiy et al., Eur. Phys. J. C 78, 15 (2018)

21. J. Benziger et al., Nucl. Instrum. Methods A582, 509 (2007)

22. M. Agostini et al., Eur. Phys. J. C 75, 506 (2015)

23. J. Janicskó Csáthy et al., Nucl. Instrum. Methods 654, 225 (2011). arxiv: 1606.04254

24. L. Baudis, G. Benato, R. Dressler, F. Piastra, I. Usoltsev, M. Walter, J. Instrum. 10, P09009 (2015)

25. R. Brugnera et al., J. Instrum. 7, P10017 (2012)

26. A. Wegmann, Dissertation. Universität Heidelberg (2017). https:// doi.org/10.11588/heidok.00022568

27. M. Heisel, Dissertation, Universität Heidelberg (2011) [URN: urn:nbn:de:bsz:16-opus-119216]

28. M. Agostini, L. Pandola, P. Zavarise, O. Volynets, J. Instrum. 6, P08013 (2011)

29. K. Freund et al., Eur. Phys. J. C 76, 298 (2016)

30. L. Baudis, A.D. Ferella, F. Froborg, M. Tarka, Nucl. Instr. Methods A 729, 557 (2013)

31. L. Baudis et al., J. Instrum. 10, P12005 (2015)

32. M.L. di Vacri et al., AIP Conf. Proc. 1672, 150001 (2015)

33. B. Lehnert, Dissertation, Universität Dresden (2016)

34. T. Goldbrunner, Dissertation, Technische Universität München (1997)

35. H.V. Klapdor-Kleingrothaus, I.V. Krivosheina, Nucl. Instrum. Methods A 566, 472 (2006)

36. M. Agostini et al. [Gerda collaboration], Eur. J. Phys. C 75, 255 (2015)

37. V. Wagner, Dissertation. Universität Heidelberg (2017). https://doi. org/10.11588/heidok.00022621

38. T. Doke et al., Nucl. Instrum. Methods 269, 291 (1988)

39. A. Hitachi, T. Takahashi, Phys. Rev. B 27, 5279 (1983)

40. P. Benetti et al., Nucl. Instrum. Methods A574, 83 (2007)

41. J. Knies, Master Thesis, University Tübingen (2016)

42. M. Agostini et al. [Gerda collaboration], Astropart. Phys. 84, 29 (2016) 